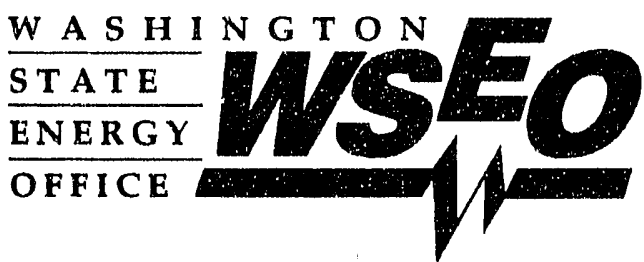

\title{
1990 Washington State Directory of Biomass Energy Facilities
}




\title{
1990 Washington State Directory of Biomass Energy Facilities
}

\author{
Prepared by \\ Joyce A. Deshaye \\ Bioenergy Program Assistant
}

(206) 586-8079

James D. Kerstetter, Ph.D. Bioenergy Program Manager

(206) 586-5034

Desktop Publishing \& Design by

Charlotte A. Gruessing

This report was prepared as an account of work sponsored by an agency of the United States Neither the United States Government nor any agency thereof, nor any of their Government. Neither the United any warranty, express or implied, or assumes any legal liability or responsiemployees, makes any warranty, express or usefulness of any information, apparatus, product, or bility for the accuracy, completeness, or usefulness of any infor process disclosed, or represents that its use would not infringe privately ow trade name, trademark, ence herein to any specific commercial product, process, or sor imply its endorsement, recommanufacturer, or otherwise does not necessarily constitute or imply its endorsernent, rhe views mendation, or favoring by the United States Government or any agency thereof. The views and opinions of authors expressed herein do not

United States Government or any agency thereof. 


\section{Acknowledgements}

The authors wish to thank the U.S. Department of Energy and the Bonneville Power Administration for their financial contribution to this effort. We also want to thank Steve Zubalik, the Washington State Energy Office, for preparing the maps included in this directory.

Special thanks are due to the facility managers that took the time to respond to the survey. Without their cooperation, this report could not have been completed.

\section{Disclaimer}

This report was prepared by the Washington State Energy Office (WSEO) as an account of work sponsored by the U. S. Department of Energy. Neither the United States, the state of Washington, the Washington State Energy Office, nor any of their employees, nor their contractors, subcontractors, nor their employees, make any warranty, expressed or implied, or assumes legal responsibility for the accuracy, completeness, or the usefulness of any information, apparatus, product, or process disclosed within this report. 


\section{Table of Contents}

Table of Abbrevlations iv

Introduction 1

Summary Table 2

Biogas Production

Biogas Facilities Map $\mathbf{3}$

Summary Table 4

Blogas Facilities 5

Listing Changes From 1987 Edition 12

Wood Combustion

Wood Combustion Facilities Map 13

Sumrnary Table 14

Forest Products Industry Facilities 16

Other Facilities $\mathbf{3 3}$

Listing Changes From 1987 Edition 36

Ethanol Fuel,

Municipal Solid Waste (MSW), and

Solld Fuel Processing

Ethanol, MSW, and Solid Fuel Facilities Map

Summary Tables $\mathbf{3 8}$

Ethanol Fuel Production Facilities 39

MSW Combustion Facilities 39

Solld Fuel Processing Facilities $\mathbf{4 0}$

Listing Changes From 1987 Edition 41

Conversicn Table 43

Glossary 45

Index 49 


\section{Table of Abbreviations}

$\begin{array}{ll}\text { BDMT } & \text { bone dry metric ton } \\ \text { BDT } & \text { bone dry ton } \\ \text { BDT/yr } & \text { bone dry ton per year } \\ \text { BDU } & \text { bone dry unit } \\ \text { bhp } & \text { boiler horse power } \\ \text { Btu } & \text { British thermal unit } \\ \text { hp } & \text { horse power } \\ \text { kW } & \text { kilowatt } \\ \text { kWh } & \text { kilowatt-hour } \\ \text { Ib/hr } & \text { pound per hour } \\ \text { lbs/hr } & \text { pounds per hour } \\ \text { MGD } & \text { million gallons per day } \\ \text { MSW } & \text { municipal solid waste } \\ \text { MW } & \text { electric capacity, megawatts } \\ \text { MWh } & \text { electric production, megawatt-hour } \\ \text { psi } & \text { pounds per square inch } \\ \text { psig } & \text { pounds per square inch, guage } \\ \text { RDF } & \text { refuse derived fuel } \\ \text { sct } & \text { standard cubic feet } \\ \text { STP } & \text { sewage treatment plant }\end{array}$




\section{Introduction}




\section{Introduction}

This is the second edition of the Washington Directory of Blomass Energy Facilities, the first edition was published in 1987. It is an update of blomass energy production and use in Washingion State for 1989. The purpose of this directory is to provide a listing of known biomass users within the state and some basic information about their facilities. The data can be helpful to persons or organizations considering the use of biomass fuels.

The directory is divided into three sections of biomass facilities with each section containing a map of locations and a data summary table. In addition, a conversion table, a glossary and an index are provided in the back of the directory.

The first section deals with biogas production from wastewater treatment plants.

The second section provides information on the wood combustion facilities in the state. This section is subdivided into two categories. The first is for facilities connected with the forest products industries. The second category include other facilities using wood for energy.

The third section is composed of three different types of biomass facilities-ethanol, municipal solid waste, and solid fuel processing.

As shown in Table 1 on page 3, biomass facilities included in this directory produce over 64 trillion Btu (British thermal units) per year. Wood combustion facilities account for 91 percent of the total. Biogas and ethanol facilities each produce close to 800 billion Btu per year, MSW facilities produce 1,845 billion Btu, and solid fuel processing facilities produce 2,321 billion Btu per year. To put these numbers in perspective, Washington's industrial sector uses 200 trillion Btu of fuels per year. Therefore, biomass fuels used and/or produced by facilities listed in this directory account for nearly 32 percent of the state's total industrial fuel demand. This is a sizable contribution to the state's energy needs.

Table 1 also shows significant increases in biomass energy production for biogas and ethanol production, MSW combustion, and solid fuel processing. 


\section{Summary Table 1 \\ Washington State Blomass Energy Production}

Comparison for 1987 and 1989

(Billion Btu - Gross)

Type of

Facllity

Blogas

Wood Combustion

Ethanol Production

MSW Combustion

Solid Fuel Processing

Total
1987

$\begin{gathered}\text { Blillon } \\ \text { Btu/Year }\end{gathered}$
600
57.496
600
300
$<1$
59,000

1989

$\%$ of

Total

1.02

97.45

1.02

0.51

0.00

$\begin{array}{rr}\begin{array}{c}\text { Billion } \\ \text { Btu/Year }\end{array} & \begin{array}{r}\text { \% of } \\ \text { Total }\end{array} \\ 810 & \\ 58,834 & 1.25 \\ 798 & 91.06 \\ 1,845 & 1.24 \\ 2,321 & 2.86 \\ 64,608 & 3.59 \\ & \end{array}$


Biogas Production 


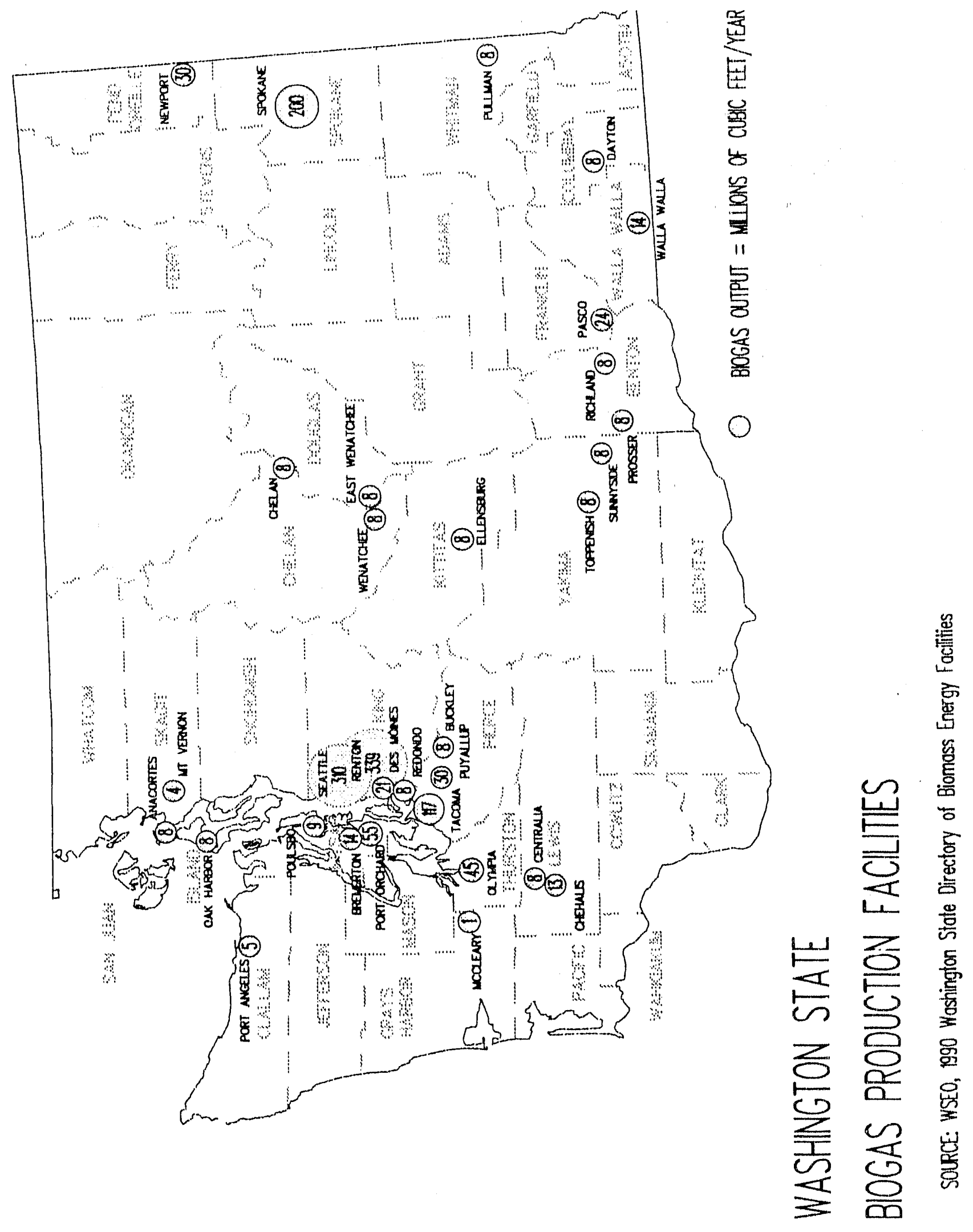




\section{Summary Table 2 \\ Washington State Blogas Production Facillties}

\section{Sorted by Blogas Output}

\begin{tabular}{|c|c|c|c|c|c|}
\hline \multicolumn{2}{|c|}{ Facllity. } & Clty & $\begin{array}{c}\text { Sowage } \\
\text { Capacity } \\
\text { Million } \\
\text { Gal./Day }\end{array}$ & $\begin{array}{l}\text { Avg. Blogas } \\
\text { Output } \\
\text { Million } \\
\text { Cublc Fu. Yr. }\end{array}$ & $\begin{array}{l}\text { Gross Energs } \\
\text { Equlvalent" } \\
\text { Milllon } \\
\text { Btu/Yr. }\end{array}$ \\
\hline$\Delta$ & Renton Treatment Plant & Renton & 72.00 & 339 & 203,400 \\
\hline a & West Point Treatment Plant & Seattle & 125.00 & 256 & 153,600 \\
\hline & Spokane Wastewater Treatment Plant & Spokane & 44.00 & 200 & 120,000 \\
\hline$\Delta$ & Tacoma STP-1, Clty of & Tacioma & 28.00 & 80 & 48,000 \\
\hline - & $\begin{array}{l}\text { Port Orchard/Kitsap County Sewer Dist. \#5 } \\
\text { Lacey, Olympla, Tumwater, Thurston }\end{array}$ & Port Orchard & 2.80 & 55 & 33,000 \\
\hline & $\begin{array}{l}\text { (LOTT) } \\
\text { Plerce Co. Chambers Creek Treatment }\end{array}$ & Olympla & 22,00 & 45 & 27,000 \\
\hline & Plant & Tacoma & 12.00 & 32 & 19,200 \\
\hline & Puyallup Wastewater Plant & Puyallup & 10.70 & 30 & 18,000 \\
\hline & Newport, City of & Newport & 0.50 & 30 & 18,000 \\
\hline & Pasco, Clty of & Pasco & 4.25 & 24 & 14,400 \\
\hline & $\begin{array}{l}\text { Des Moines Creek Treatment Plant } \\
\text { SW Suburban Sewer District - }\end{array}$ & Des Moines & 6.00 & 21 & 12,600 \\
\hline & Salmon Creek & Seattle & 5.80 & 15 & 9,000 \\
\hline & SW Suburban Sewer District - Miller Creek & Seattle & 4.70 & 15 & 9,000 \\
\hline & METRO/Alki Treatment Plant & Seattle & 15.00 & 15 & 9,000 \\
\hline & Walla Walla Wastewater Treatment Plant & Walla Walla & 15.00 & 14 & 8,400 \\
\hline . & Bremerton, Clty of & Bremerton & 15.00 & 14 & 8,400 \\
\hline & Chehalis, City of & Chehalls & 2.00 & 13 & 7,800 \\
\hline & p Treatment Plant & Poulsbo & 4.80 & 9 & 5,400 \\
\hline & Ellensbur & Ellensberg & 15.00 & 8 & 4,800 \\
\hline & or \& Sewer & Redondo & 13.80 & 8 & 4,800 \\
\hline & Wenatchee Water Pollution Control Plant & Wenatchee & 5.00 & 8 & 4,800 \\
\hline m & Centralla, City of & Centralia & 4.30 & 8 & 4,800 \\
\hline & Anaco & Anacortes & 2.20 & 8 & 4,800 \\
\hline & ide Wastewater Treatment Plant & Sunnyside & 2.18 & 8 & 4,800 \\
\hline & Douglas County Sewer District & East Wenatchee & 1.60 & 8 & 4,800 \\
\hline & Prosser, City of & Prusser & 1.50 & 8 & 4,800 \\
\hline & Toppenish Wastewater Treatment Plant & Toppenish & 1.35 & 8 & 4,800 \\
\hline & Treatment Plant & Chielan & 1.20 & 8 & 4,800 \\
\hline & Oak Harbor, Clty of & Oak Harbor & 0.85 & 8 & 4,800 \\
\hline & Dayton, Clty of & Dayton & 0.60 & 8 & 4,800 \\
\hline & Raínier State School & Buckley & NA & 8 & 4,800 \\
\hline & Pullman Wastewater Treatment Plant & Pullman & 4.30 & 8 & 4,800 \\
\hline m & Richland Wastewater Treatment Plant & Richland & 8.90 & 8 & 4,800 \\
\hline & Tacome & Tacoma & 10.00 & 5 & 3,000 \\
\hline & Port Angeles, City of & Port Angeles & 3.20 & 5 & 3,000 \\
\hline & Richmond Beach Treatment Plant & Seattle & 3.20 & 5 & 3,000 \\
\hline & Carkeek Park Treatment Plant & Seattle & 3.50 & 4 & 2,400 \\
\hline & Mount Vernon, City of & Mount Vernon & 4.50 & 4 & 2,400 \\
\hline & McCleary Sewage Treatment Plant & McCleary & 0.75 & $<1$ & 276 \\
\hline & ( & & 77.48 & $\overline{1,350}$ & 0.276 \\
\hline
\end{tabular}

NA - Information Nri Avallable

"Calculated at 600 Btu per scf Instead of 1,000 Btu reported for this facility

**Average output calculated at 600 Btu per cublc foot

***Calculated by WSEO

- Supplies Washington Natural Gas Pipeline

- Electrical generatlon capacity 


\section{Biogas Facilities}

\author{
Anacortes Publlc Works/Operations \\ Division, City of \\ 2201 - 37th Street \\ Anacortes, WA 98221 \\ Phone: (206) 293-5175 \\ Contact: Mike Foster \\ Plant Design Capacity: \\ Sewage (MGD): $2.2^{* *}$ \\ Electrical Generation (MW): None
}

Average Plant Output:

Biogas (cubic feet): Less then 10 million per

year

Electrical Generation (MW): None

End Use(s): Space and process heat

* Presently in pre-design phase for secondary treatment. Final design capacity unknown at this time.

\author{
Bremerton Wastewaier Treatment Plant, \\ City of \\ Bremerton, WA \\ Phone: (206) 479.4646 \\ Contact: Pat J. Coxon \\ Plant Design Capacity: \\ Sewage (MGD): 15 \\ Electrical Generation (MW): $140 \mathrm{~kW}$ \\ Average Plant Output: \\ Blogas (cubic feet): \\ Electrical Generation (MW): $70 \mathrm{kWh}$
}

End Use(s): Process heat

\section{Central KItsap Treatment Plant \\ 12350 State 303 NE \\ Poulsbo, WA 98370 \\ Phone: (206) 876-7197 \\ Contact: Ralph DeClements \\ Plant Design Capacity: \\ Sewage (MGD): 4.8 \\ Electrical Generation (MW): None}

Average Plant Output:

Biogas (cubic feet): $9,320,000$ per year

Electrical Generation (MW): None

End Use(s): Space and process heat
Centralla Wastewater Treatment Plant, Clty of

1401 W Mellen Street

Centralia, WA 98531

Phone: (206) 736-2853

Contact: Denlse Lahmann, Administrator, or Ron Davis, Manager

Plant Design Capacity: Sewage (MGD): 4.3 Electrical Generation (MW): Standby generator $250 \mathrm{~kW}$ three phase, 277/480 volts

Average Plant Output: Biogas (cubic feet): Less than 10 million per year (anaerobic digestion)

Electrical Generation (MW): None

End Use(s): Space and process heat

\section{Chehalls Wastewater Treatment Plant,} City of

1191 NW Shoreline Drive

Chehalis, WA 98532

Phone: (206) 748-7187

Contact: Robert Pinkerton

Plant Design Capacity: Sewage (MGD): 2.0 (average dry weather)

Electrical Generation (MW): None

Average Plant Output:

Blogas (cubic feet): Less than 15 million per year

Electrical Generation (MW): None

End Use(s): Process heat

\section{Chelan Treatment Plant}

$S$ Robinson Street

Chelan, WA 98816

Phone: (509) 682-2211

Contact: Howard Merchant

Plant Design Capacity: Sewage (MGD): 1.2 (new R.B.C. plant) Electrical Generation (MW): None

Average Plant Output: Unknown Blogas (cubic feet): Less than 10 million per year Electrical Generation (MW): None

End Use(s): Flared 


\author{
Dayton Wastewater Treatment Plant, \\ Clty of \\ Route 1 \\ Dayton, WA 99328 \\ Phone: (509) 382-2937 \\ Contact: Duane Lowe \\ Plant Design Capacity: \\ Sewage (MGD): 0.60 \\ Electrical Generation (MW): None \\ Average Plant Output: \\ Biogas (cubic feet): Less than 10 million per \\ year \\ Electrical Generation (MW): None
}

End Use(s): Srace heat, flared, automatic burn off

\section{Des Moines Creek Treatment Plant 1200 S 216th Street \\ Des Moines, WA 98198 \\ Phone: (206) 824-2760 \\ Contact: Clint Read, Superintendent \\ Plant Design Capacity: Sewage (MGD): 6.0 Electrical Generation (MW): None}

\section{Average Plant Output: \\ Biogas (cubic feet): 60,000 per day, $20-22$ million per year Electrical Generation (MW): None}

End Use(s): Space and process heat

\section{Douglas County Sewer Dlstrlct}

P.O. Box 7129

East Wenatchee, WA 98801

Phone: (509) 884-2484

Contact: Dale G. O'Neal

Plant Design Capacity: Sewage (MGD): 1.6 Electrical Generation (MW): None

Average Plant Output: 1 MGD Biogas (cubic feet): Less than 10 million per year Electrical Generation (MW): None

End Use(s): Process heat

\section{Ellensburg Wastewater Treatment} Plant, Clty of

Route 2, Box 13

Ellensburg, WA 9892.6

Phone: (509) 962-7277

Contact: Stan Miller

Plant Design Capacity:

Sewage (MGD): 15

Electrical Generation (MW): None

Average Plant Output:

Biogas (cubic feat): Less than 10 million per year

Electrical Generation (MW): None

End Use(s): Process heat and digester mixing

Federal Way Water \& Sewer Redondo Treatment Plant

515 S 288th Street

Redondo, WA 98054

Phone: (206) 839-744r

Contact: Chuck Babel, Assistant Supervisor

Plant Design Capacity:

Sewage (MGD): 4.32 average

annual, 13.80 peak hydraulic, and 5.47

average wet weather

Electrical Generation (MW): None

Average Plant Output:

Biogas (cubic feet): Less than 10 million per year

Electrical Generation (MW): Nont

End Use(s): Space and process heat

Lacey, Olympla, Tumwater, Thurston

County (LOTT) Wastewater

Treatment Plant

$500 \mathrm{~N}$ Adams Street

Olympia, WA 98501

Phone: (206) 753-8386

Contact: Tom Kolby

Plant Design Capacity:

Sewage (MGD): 22

Electrical Generation (MW): Back-up 
Average Plant Output:

Blogas (cubic feet): 44.8 million per year Electrical Generation (MW): 1,307,642 kWh

End Use(s): Power generation and process heat

\author{
McCleary Sewage Treatment Plant \\ Maple Street \\ McCleary, WA 98557 \\ Phone: (206) 495-3217 \\ Contact: Jim Wright \\ Plant Design Capacity: \\ Sewage (MGD): 0.75 max., 0.25 avg., $0.15 \mathrm{~min}$. \\ Electrical Generation (MW): None \\ Average Plant Output: \\ Biogas (cubic feet): Less than 1 million per year \\ Electrical Generation (MW): None
}

End Use(s): Process heat

\author{
Mount Vernon Sewer Department, City of \\ 1401 Britt Road \\ Mount Vernon, WA 98273 \\ Phone: (206) 336-6219 \\ Contact: Chuck Saunders \\ Plant Design Capacity. \\ Sewage (MGD): 4.5 \\ Electrical Generation (MW): None \\ Average Plant Output: \\ Blogas (cubic feet): 4 million per year \\ Electrical Generation (MW): None
}

End Use(s): Space heat and process heat

\author{
Newport Wastewater Treatment Plant, \\ Clty of \\ 5200 Washington Street \\ Newport, WA 99156 \\ Phone: (509) 447-3012 \\ Contact: Raymond D. King \\ Plant Design Capacity: \\ Sewage (MGD): 0.5 \\ Electrical Generation (MW): None \\ Average Plant Output: \\ Biogas (cubic feet): 30 million per year \\ Electrical Generation (MW): None
}

End Use(s): Process heat
Oak Harbor, Clty of

3075 - 300 Avenue W

Oak Harbor, WA 98277

Phone: (206) 679-5551, ext. 230

Contact: Jim Croft

Plant Design Capacity:

Sewage (MGD): .85

Electrical Generation (MW): None

Average Plant Output:

Biogas (cubic feet): Less than 10 million

per year

Electrical Generation (MW): None

End Use(s): Process heating. Digested sludge is used as fertilizer.

Pasco Sewage Treatment Plant, Clty of

D \& Grey Streets

P.O. Box 293

Pasco, WA 99301

Phone: (509) 545-3468

Contact: Harold Posthuma

Plant Design Capacity:

Sewage (MGD): 4.25

Electrical Generation (MW): None

Average Plant Output:

Biogas (cubic feet): 65,000 per day

Electrical Generation (MW): None

End Use(s): Space and process heat

\section{Port Angeles, Clty of}

1509 E Columbia Street

Port Angeles, WA 98362

Phone: (206) 451-0411, ext. 165

Contact: Jim Abernathy

Plant Design Capacity:

Sewage (MGD): 3.2

Electrical Generation (MW): None

Average Plant Output:

Biogas (cubic feet): 5,000,000 per year

Electrical Gerreration (MW): None

End Use(s): Process heat 


\section{Port Orchard/KItsap County Sewer District No.5 \\ Wastewater Treatment Facllity \\ 1165 Beach Drive \\ Port Orchard, WA 98366 \\ Phone: (206) 895-2440 \\ Contact: Douglas W. Martin, Plant Manager \\ Plant Design Capacity: \\ Sewage (MGD): 2.8 \\ Electrical Generation (MW): None}

Average Plant Output: 1.2 MGD

Blogas (cubic feet): $50-60$ million per year

Electrical Generation (MW): None

End Use(s): Space and process heat, digester mixirig

\section{Prosser Wastewater Treatment Facillties, Clty of \\ Box 271 \\ Prosser, WA 99350 \\ Phone: (509) 786-3831 \\ Contact: Walter Titus \\ Plant Design Capacity: Sewage (MGD): 1.5 (wastewater) Electrical Generation (MW): None \\ Average Plant Output: \\ Biogas (cubic feet): Less than 10 millic. per year \\ Electrical Generation (MW): None}

End Use(s): Process heat

\section{Pullman Wastewater Treatment Plant} 1025 Guy Street

Pullman, WA 99163

Phone: (509) 334-4555, ext. 233

Contact: Terry Dokken

Plant Design Capacity:

Sewage (MGD): 4.3

Electrical Generation (MW): None

Average Plant Output:

Biogas (cubic feet): 7,839,000 per year

Electrical Generation (MW): None

End Use(s): Space and process heat

\section{Puyallup Wastewater Plant}

218 W Ploneer Street

Puyallup, WA 98371

Phone: (206) 841-5511

Contact: B. Michas,i Webb

Plant Design Capacity:

Sewage (MGD): 10.7

Electrical Generation (MW): None

Average Plant Output:

Biogas (cubic feet): $10-50$ million per year

Electrical Generation (MW): None

End Use(s): Space and process heal. Sludge is used as compost mixed with sawdust and used by nurseries for flowering plants and trees.

\section{Ralnler State School Sewage \\ Treatment Plant}

P.O. Box 600

Buckley, WA 98321

Phone: (206) 829-1734, ext. 280

Contact: Roy Campbell

Plant Design Capacity: Sewage (MGD):

Electrical Generation (MW): None

Average Plant Output:

Blogas (cubic teet): Less than 10 million per year

Electrical Generation (MW): None

End Use(s): Heating the digester, supplemented with natural gas

\section{Renton Treatment Plant}

1200 Monster Road SW

Renton, WA 98055

Phone: (206) 684-2412

Contact: Dick Finger, Process Control Supervisor

Plant Design Capacity: Sewage (MGD): 72

Electrical Generation (MW): None

Average Plant Output:

Biogas (cubic feet): 560,000@1,000

Btu/SCF

Electrical Generation (MW): None

End Use(s): Pipeline gas 
Blchland Wastewater Treatment Plant

P. O. Box 190

Richland, WA 99352

Phone: (509) 943-9161, ext. 416

Contact: David Nelsen

Plant Design Capacity: Sewage (MGD): 8.9 average flow

Electrical Generation (MW): $95 \mathrm{~kW}$ continuously, May-October

Average Plant Output:

Biogas (cubic feet): 21,000 per day

Electrical Generation (MW): None

End Use(s): Space and process heat

\section{Seattle - Carkeek Park Treatment Plant 1201 NW Carkeek Fark Road \\ Seattle, WA 98177 \\ Phone: (206) 684-1800 \\ Contact: Bill Berry \\ Plant Design Capacity: Sewage (MGD): 3.5 \\ Electrical Generation (MW): None \\ Average Plant Output: \\ Biogas (cubic feet): 4.2 million per year \\ Electrical Generation (NW): None}

End Use(s): Space and process heat

\author{
Seattle - Metro Alk! Treatment Plant \\ 3380 Beach Drive SW \\ Seattle, WA 98116 \\ Phone: (206) 684-2412 \\ Contact: Dick Finger \\ Plant Design Capacity: \\ Sewage (MGD): 15 \\ Eleutrical Generation (MW): None \\ Average Plant Output: \\ Biogas (cubic feet): 40,000 per day, 14.6 million \\ per year \\ Electrical Generation (MW): None \\ End Use(s): Space and process heat
}

\section{Seattle - Mlller Creek Sewage}

Treatment Plant

Southwest Suburban Sewer District

1015 SW 174th Street

Seattle, WA 98166

Phone: (206) 244-2202

Contact: Tim Yokers

Plant Design Capacity: Sewage (MGD): 4.7 (peak month)

Electrical Generation (MW): None

Average Plant Output:

Blogas (cubic feet): 42,000 per day

Electrical Generation (MW): None

End Use(s): Space and process heat, excess flared off

\section{Seattle - Rlchmond Beach Treatment}

Plant

20001 Richmond Beach Drive NW

Seattle, WA 98177

Phone: (206) 546-2826

Contact: Bill Berry

Plant Design Capacity:

Sewage (MGD): 3.2

Electrical Generation (MW): None

Average Plant Output:

Biogas (cubic feet): 5 million per year

Electrical Generation (MW): None

End Use(s): Space and process heat

\section{Seattle - Salmon Creok Sewage Treatment Plant}

Southwest Suburban Sewer Distrlct

12550 Shorewood Drive SW

Seattle, WA 98146

Phone: (206) 243-7770

Contact: Phil Baga

Plant Design Capacity:

Sewage (MGD): 5.8 (peak

month)

Electrical Genera.ion (MW): None 
Average Plant Output:

Blogas (cublc feet): 42,000 per day

Electrical Generation (MW): None

End Use(s): Process and building heat, exoess flared off

Seattle - West Polnt Treatment Plant 821 Second Avenue

Seattle, WA 98104

Phone: (206) 477-6801

Contact: Gordon Gabrielson

Plant Design Capacity:

Sewage (MGD): 125

Electrical Generation (MW): Gas production is no longer adequate to power three $1.3 \mathrm{MW}$ generator. Gas production should be adequate again in 1996

Average Plant Output:

Biogas (cublc teet): 700,000 per day

Electrical Generation (MW): 1.3

End Use(s): Space and process heat, electricity, equipment drives

Note: In 1988, solids were transferred to the Renton Treatment Plant reducing the biomass available for digesters by nearly $50 \%$ creating a corresponding reduction in biogas.

\section{Spokane Wastewater Treatment Plant \\ N 4401 White Park Way \\ Spokane, WA 99205 \\ Phone: (509) 456.4305 \\ Contact: Ray D. King \\ Plant Design Capacity: \\ Sewage (MGD): 44,000,000 \\ Electrical Generation (MW): None}

Average Plant Output:

Biogas (cubic feet): $100-300$ million

Electrical Generation (MW): None

Eno Use(s): Space and process heat, air conditioning, absorption chiller

\section{Sunnyside Wastewater Treatment}

Plant

$S$ Fourth Street

Sunnyside, WA 98944

Phone: (509) 837-5206

Contact: Charles Kennedy

Plant Design Capacity:

Sewage (MGD): 2.18

Electrical Generation (MW): None

Average Plant Output:

Biogas (cubic feet): Less than 10 million per year

Electrical Generation (MW): None

End Use(s): Process heat

\section{Tacoma - Plerce County Chambers}

Creek Treatment Plant

10311 Chamber Creek Road

Tacoma, WA 98467

Phone: 565-3013

Contact: Steve Thompson, Lab Supervisor

Plant Design Capacity: Sewage (MGD): 12

Electrical Generation (MW): None

Average Plant Output: $8.5 \mathrm{MGD}$ Biogas (cubic feet): $32,665,800$ per year Electrical Generation (MW): None

End Use(s): Space and process heat, heat digesters

Tacoma Sewage Treatment Plant-1,

\section{Clty of}

2201 Portland Avenue

Tacoma, WA 98421

Phone: (206) 591.5587

Contact: David Hufford

Plant Design Capacity: Sewage (MGD): 28

Electrical Generation (MW): None

Average Plant Output:

Biogas (cubic feet): 80 million per year Electrical Generation (MW): None

End Use(s): Space and process heat, hot water, autos, Washington Natural Gas pipeline 
Tacoma Sewage Treatment Plant-3,

\section{Clty of}

4002 Waterview Street

Tacoma, WA 98507

Phone: (206) 591-5587

Contact: David Hutford

Plant Design Capacity:

Sewage (MGD): Less than 10

Electrical Generation (MW): None

Average Plant Output:

Blogas (cubic feet): 5 million per year

Electrical Generation (MW): None

End Use(s): Space and process heat

Toppenish Wastewater Treatment Plant Germantown Road

Toppenish, WA 98948

Phone: (509) 865-4089

Contact: Ed Martindale

Plant Design Capacity:

Sewage (MGD): 1.35

Electrical Generation (MW): None

Average Plant Output:

Blogas (cubic feet): Less than 10 million per year

Electrical Generation (MW): None

End Use(s): Process heat

\section{Walla Walla Wastewater \\ Treatment Plant \\ 571 Hatch Street \\ Walla Walla, WA 99362 \\ Phone: (509) 529-2859 \\ Contact:}

Plant Design Capacity:

Sewage (MGD): 10.8 dry flow,

15.00 wel flow

Electrical Generation (MW): None

Average Plant Output:

Biogas (cubic feet): $14,235,000$ per year Electrical Generation (MW): None

End Use(s): Space and process heat, $8,395,000$ cubic feet

\section{Wenatchee Water Pollution}

Control Plant

201 N Worthen Street

P.O. Box 519

Wenatchee, WA 98801

Phone: (509) 663-8416

Contact: Mel Heckler

Plant Design Capacity:

Sewage (MGD): 5

Electrical Generation (MW): None

Average Plant Output:

Blogas (cubic teet): Less than 10 million per year

Electrical Generation (MW): None

End Use(s): Process heat 


\section{Blogas Production Faclitiles}

\section{Listing Changes \\ From 1987 Edition}

\begin{tabular}{|c|c|c|}
\hline \multicolumn{2}{|l|}{ New Llstlngs } & Locatlon \\
\hline \multicolumn{2}{|c|}{ Des Moines Creek Treatment Plant } & Des Moines \\
\hline \multicolumn{2}{|c|}{ Port Orchard/Kitsap County Sewer District \#5 } & Port Orchard \\
\hline \multicolumn{2}{|c|}{ Southwest Suburban Sewer District - Miller Creek } & Seattle \\
\hline \multicolumn{2}{|c|}{ Southwest Suburban Sewer District - Salmon Creek } & Seattle \\
\hline Deletions & Reason & Location \\
\hline City of Grand Coulee & No biogas production & Grand Coulee \\
\hline City of Tacoma STP-2 & Facility closure & Tacoma \\
\hline
\end{tabular}




\section{Wood Combustion - Forest Products Industry Facilities - Other Facilities}


(렁
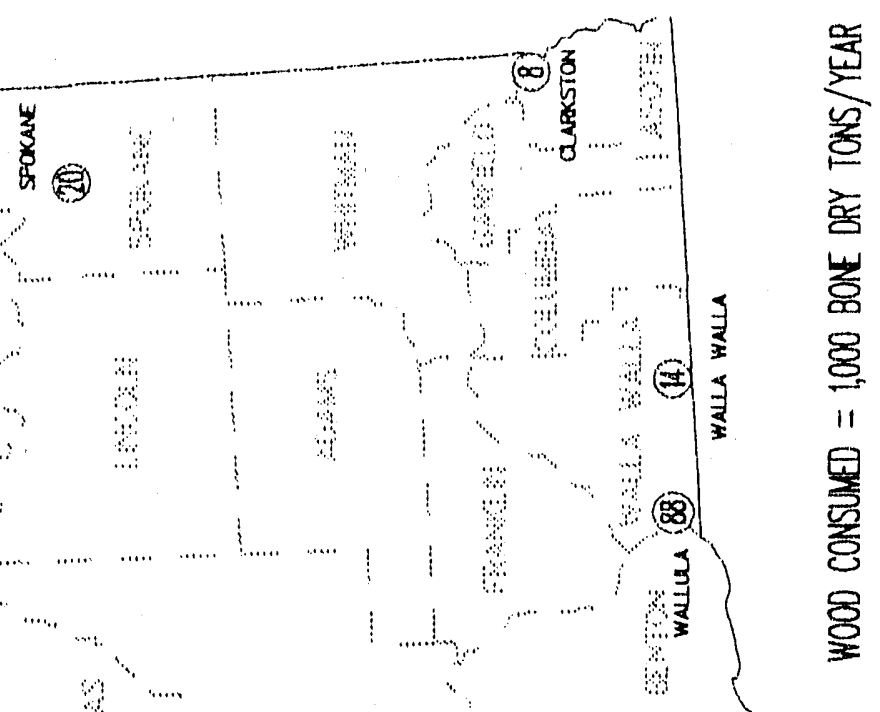

(2)
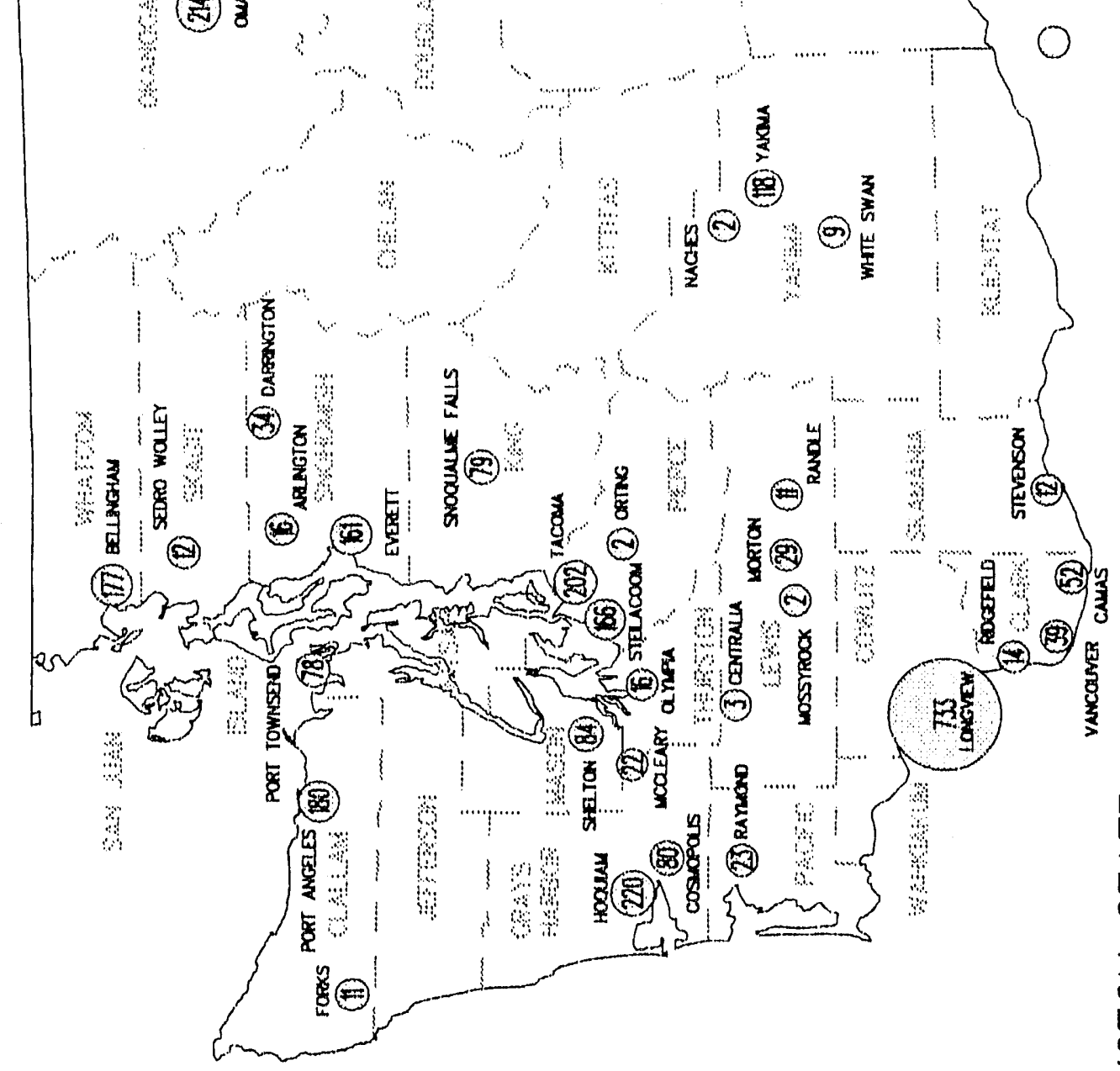


\section{Summary Table 3 \\ Washington State Wood Combustion \\ Facillties Sorted by Wood Consumption}

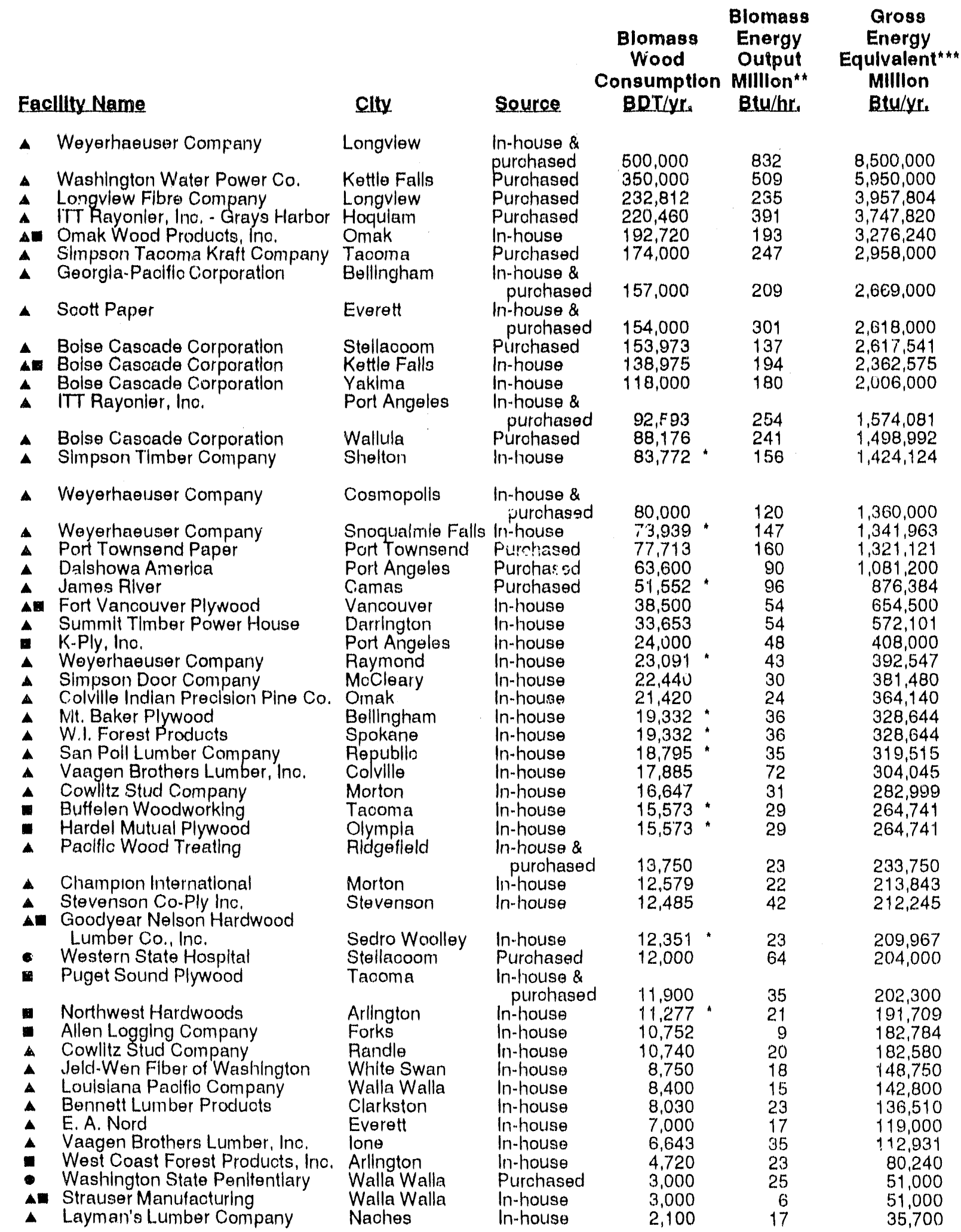




\section{Summary Table 3}

(ContInued)

\section{Faollity Name}

- Maple Lane Sohool

- Washlngton Soldiers Home \&

$$
\text { Colony }
$$

- Degoede Bulb Farms

- Klnnear of Washington

- Oeser Company. The

- Fred Tebb \& Sons, Inc.

- Coestoraft, Incorporated

- Plum Creok Manufaoturing, Ino.

A Smlth Streot Mill, Ino.

Total

\section{clty}

Centralla

Orting

Mossyrook

Contralia

Bellingham

Tacoma

Taooma

Colville

Everett

Blomass Type:

$\triangle$ Hog Fuel

- Mill Residue

- Wood Palets

NA. Information not avallable

"Estlmated by Washington State Energy Offloe

* Caloulated from reported average steam output

*" Caloulated at 8,500 Btu per pound of blomass wood oonsumed 


\section{Forest Products Industry Facilities}

\author{
Allen Logging Company \\ HC 80, Box 736 \\ Forks, WA 98331 \\ Phone: (206) 374-6000 \\ Contact: Gerry Lane
}

Type of Business: Lumber and veneer

Type of Blomass Fuel: Mill residue

Source of Fuel: In-house

Annual Blomass Fuel Consumed: 15,360 green lons

Back-up Fuel: None

End Use(s): Klln dryers

Plant Design Capaoity:

Heat (million Btu/hr):

Steam:10,000 lbs/hr@110psig

Eleotrical Generation (MW): None

Average Plant Output:

Heat (million Btu/hr):

Steam:7,500lbs/hr @110 psig

Electrical Generation (MW): None

Type of Combustor/Furnace: Fuel cell

Manufacturer: Wellons

Type of Boller: Erie water tube - modifled VL

Boiler Manufacturer: Erie

Type of Pollution Control: Multicone

Reason for Using Wood Fuel: Cost, avallability

\section{Bennett Lumber Products}

1785 Elm Street

Clarkston, WA 99403

Phone: (509) 758-5558

Contact: Don Fairley

Type of Business: Lumber

Type of Blomass Fuel: Hog fuel

Source of Fuel: In-house

Annual Blomass Fuel Consumed: 7,300 units

Back-up Fuel: None

End Use(s): Head for dry kilns

Plant Design Capacity:

Heat (million Btu/hr): 22,425,000

Steam: $19,500 \mathrm{lbs} / \mathrm{hr} @ 100 \mathrm{psig}$

Electrical Generation (MW):
Average Plant Output:

Heat (million Btu/hr): $2,070,000$

Steam: $8,000 \mathrm{lbs} / \mathrm{hr} @ 100 \mathrm{psig}$

Eleotrical Generatlon (MW):

Type of Combusterirurnace: Water tube

Manuiacturer: Kipper

Type of Boller: Water tube

Boiler Manufacturer: Kipper

Type of Pollution Control: Multicone \& eleotric preolpltator

Feason for Using Wood Fuel: Generated during manufacturing

\section{Bolse Cascade Corporation}

Kettle Falls Lumber - Steam Boller

P.O. Box 310

Keitle Falls, WA 99141

Phone: (509) 738-6421

Contact: Rod Smith

Type of Business: Lumber

Type of Blomass Fuel: Hog fuel, planner shavings and sawdust

Source of Fuel: In-house

Annual Blomass Fuel Consumed: 57,451 green tons

Back up Fuel: None

End Use(s): Diy kilns and unit heaters

Plant Design Capaclty:

Heat (million Btu/hr):

Steam: 50,000 lbs/hr@ 125 psig

Electrical Generation (MW):

Average Plant Output:

Heat (million Btu/hr):

Steam: 35,700 lbs/hr@125 psig

Electrical Generation (MW):

Type of Combuster/Furnace: 3 Cell CycloBlast

Manufacturer: Wellons

Type of Boller: Water tube

Boller Manufacturer: Nebraska Boller Company

Type of Pollutlon Control: Dry type mechankcal multicone collector

Reason for Using Wood Fuel: Economics 


\section{Bolse Cascade Corporation \\ Kettle Falls Plywood - Fluld \\ Bed Burners \\ P.O. Box 310 \\ Kettle Falls, WA 99141 \\ Phone: (509) 738-6421 \\ Contact: Rod Smith}

Type of Business: Plywood

Type of Blornass Fuel: Hog fuel, sawdust

Source of Fuel: in-house

Alinual Blomass Fuel Consumed: 95,000 green tons

Back-up Fuel: Gas

End Use(s): Plywood veneer dryers (direct heat souroe)

Plant Design Capacity:

Heat (milllion Btu/hr): 160

Stoam:_lbs/hr@

Electrical Generation ( $\overline{\mathrm{MW}}$ ): psig

Average Plant Output:

Heat (million Btu/hr): 80

Steam:__lbs/hr@

Electilcal Generation (MW): psig

Type of Combuster/Furnace: Fluid bed

Manufacturer: Energy Products of Idaho (E.P.I.)

Type of Boller: None

Boiler Manufacturer:

Type of Pollution Control: $6^{n}$ Multicone collector \& wet scrubber

Reason for Using Wood Fuel: Economics

\section{Bolse Cascade Corporation \\ Kettle Falls Plywood \& Small Log Mill - Steam Boller \\ P.O. Box 310 \\ Kettle Falls, WA 99141 \\ Phone: (509) 738-6421 \\ Contact: Rod Smith}

Type of Business: Lumber \& plywnod

Type of Blomass Fuel: Hog fuel, sawdust \& plywood dry trim

Source of Fuel: In-house

Annual Biomass Fuel Consumed: 46,084 green tons

Back-up Fuel: None

End Use(s): Steam vats, plywood hot press, unit heaters
Plant Design Capacity:

Heat (million Btu/hr):

Steam: 60,000 Ibs/hr @ $125 \mathrm{psig}$

Electrical Generation (MW):

Average Plant Output:

Heat (million Btu/hr):

Steam: $28,640 \mathrm{lbs} / \mathrm{hr}$ (c) $125 \mathrm{psig}$

Electrical Generation (MW):

Type of Combuster/Furnace: Fleld erected stationary water cooled grate

Manufacturer: Riley Stoker Corporation

Type of Boiler: Water lube, type "VO"

Boller Manufacturer: Rilley Stoker Corporation

Type of Pollution Control: Dry type 9" Mechanical Multioone Collector

Reason for Using Wood Fuel: Economics

\section{Bolse Cascade Corporation}

4302 Chamber Creek Road

Siellacoom, WA 98388

Phone: (206) 588-2115

Contact: Ken Campbell

Type of Business: Pulp and paper

Type of Blomass Fuel: Hog fuel

Source of Fuel: Various lumber companies

(all purchased)

Annual Blomass Fuel Consumed: 153,973

BDT hogged fuel plus 17,472 tons sludge primary clarifier

Back-up Fuel: Industrial \#6 oll

End Use(s): Process steam, dryers

Plant Design Capacity: No 5 Hogged fuel boller

Heat (million Btu/hr): None

Steam: $150,000 \mathrm{lbs} / \mathrm{hr} @ 140 \mathrm{psig}$

Electrical Generation (MW): None

Average Plant Output:

Heat (million Btu/hr):

Steam: 120,000 lbs/hr@140psig

Electrical Generation (MW): None

Type of Conibuster/Furnace:

Manufacturer: Kipper \& Sons

Type of Boiler: Traveling grate, water wall

Boller Manufacturer: Kipper \& Sons

Type of Pollution Control: Multicone collectors/fly ash reinjection, AER POL high energy wei scrubber 
Reason for Using Wood Fuel: Cost/avallability vs fossil fuel Also firm is equipped with a Cas. cade (Bacho design) fuel dryer utllizing flue gas

\section{Bolse Cascade Corporation}

P.O. Box 500

Wallula, WA 99363

Phone: (509) 545-3213

Oontact: Anders Ohlsson, Production Manager

Type of Business: Pulp and paperboard

Type of Blomass Fuel: Hog fuel

Source of Fuel: Purchased

Annual Blomass Fuel Consumed: 73,480 BDUs

Back-up Fuel: Natural gas

End Use(s): Process steam, dryers

Plant Design Capacity: 1 hog fuel boller

Heat (million Btu/hr): 193

Steam: 201,000 lbs/hr@250 psig

Electrical Generation (MW): None

Average Plant Output:

Heat (million Btu/hr): 172

Stoarn: 179,000 lbs/hr @ 250 psig

Electrical Generation (MW): None

Type of Combuster/Furnace: Sterling type, pin hole grate

Manufacturer: Kipper

Type of Boller: Sterling type pinthold grate

Boller Manufacturer: Klpper

Type of Pollution Control: Mechanical, multicone scrubber, Rilley ventri-rod wet scrubber

Reason for Using Wood Fuel: Economics

\section{Bolse Cascade Corporation}

\section{P.O. Box 51}

Yakima, WA 98901

Phone: (509) 453-3131

Contact: Dick Godfrey

Type of Business: Lumber

Type of Blomass Fuel: Hog fuel

Source of Fuel: In-house

Annual Blomass Fuel Consumed: 118,000 dry tons

Back-up Fuel: None

End Use(s): Dry kilns, dryers
Flant Design Capacity:

Heat (million Btu/hr):

Steam: $200,000 \mathrm{lbs} / \mathrm{hr} @ 250 \mathrm{psig}$

Electrical Generation (MW): None

Average Plant Output:

Heat (millicn Btu/hr):

Steam: 150,000 lbs/hr@250 psig

Electrical Generation (MW):

Type of Combuster/Furnace: 3 Dutch ovens, 1 spreader stoker

Manufacturer:

Type of Boller: Water tube

Boller Manufacturer:

Type of Pollution Control: Multicone and wet sorubber

Reason for Using Wood Fuel: Economlcs

\section{Buffelen Woodworking}

P.O. Box 1383

Tacoma, WA 98481

Phone: (206) 627-1191

Contact: Roger Lay

Type of Business: Door

Type of Blornass Fuel: Mill residue

Source of Fuel: In-house

Annual Blomass Fuel Consumed:

Back-up Fuel: Propane

End Use(s): Dry kiln

Plant Design Capacity:

Heat (million Btu/hr):

Steam: 55,000 ibs/hr@_psig

Electrical Generation (MW): None

Average Plant Output:

Heat (million Biu/hr):

Steam:25,000lbs/hr@

Electrical Generation (MW): psig

Type of Combuster/Furnace:

Manufacturer:

Type of Boiler: Water tube

Boiler Manufacturer: Riley Stoker

Type of Pollution Control: Wet scrubber

Reason for Using Wood Fuel: Cost, availability 


\section{Champlon International \\ Drawer A \\ Morton, Washington 98356 \\ Phone: (206) 496-6333 \\ Contact:}

Type of Business: Lumber, veneer

Type of Biomass Fuel: Hog fuel

Source of Fuel: In-house

Annual Biomass Fuel Consumed: 11,435 units

Back-up Fuel: None

End Use(s): Dry kilns, hot water vats

Plant Design Capacity: 20,000

Heat (million Btu/hr): 23.1

Steam: 20,000 lbs/hr@125 psig

Electrical Generation (MW): None

Average Plant Output:

Heat (million Btu/hr): 21.2

Steam: 18,333 lbs/hr @ 125 psig

Electrical Generation (MW): None

Type of Combuster/Furnace: Dutch oven

Manufacturer: Zinda

Type of Boiler: Double drum, water tube, type RO

Boiler Manufacturer: Riley

Type of Pollution Control: Ash separator, Lear Sigler Monitor

Reason for Using Wood Fuel: Economics

\section{Colville Indlan Precision PIne Company \\ P.O. Box 3293 \\ Ornak, Washington 98841 \\ Phone: (509) 826-1921 \\ Contact: Dan Brudevold}

Type of Business: Lumber

Type of Biomass Fuel: Hog fuel

Source of Fuel: In-house

Annual Biomass Fuel Consumed: 21,420 BDT

Back-up Fuel: None

End Use(s): Dry kilns

Plant Design Capacity:

Heat (million Btu/hr):

Steam:_اbs/hr@

Electrical Generation ( $\overline{\mathrm{MW}}$ ):
Average Plant Output:

Heat (million Btu/hr):

Steam: 21,000 lbs/hr @

Electrical Generation ( $\mathrm{MW}$ ): psig

Type of Combuster/Furnace:

Manufacturer: Wellons

Type of Boiler: 20,000 lbs/hr single cell

Boiler Manufacturer: Wellons

Type of Pollution Control: Dry scrubber

Reason for Using Wood Fuel: Economics

\section{Coastcraft, \|ncorporated}

1002 E "F" Street

Tacoma, WA 98421

Phone: (206) 272-1154

Contact: Bill Rigby

Type of Business: Woodwork, windows, doors

Type of Blomass Fuel: Mill residues, sawdust

Source of Fuel: In-house

Annual Biomass Fuel Consumed:

Back-up Fuel: None

End Use(s): Dry kiln \& heating

Plant Design Capacity:

Heat (million Btu/hr):

Steam: $500 \mathrm{lbs} / \mathrm{hr} @$ ___ psig

Electrical Generation (MW):

Average Plant Output:

Heat (million Btu/hr):

Steam: Ibs/hr@

Electrical Generation (MW):

Type of Combuster/Furnace:

Manufacturer:

Type of Boiler: Fire tube

Boiler Manufacturer:

Type of Pollution Control: None

Reason for Using Wood Fuel: By-product

\section{Cowlltz Stud Company}

P.O. Box 219

Randle, WA 98377

Phone: (206) 497-5030

Contact: Lloyd Cook or Charlie Allen

Type of Business: Lumber

Type of Biomass Fuel: Hog fuel 
Source of Fuel: In-house

Annual Blomass Fuel Consumed:

Back-up Fuel: None

End Use(s): Dry kilns

Plant Design Capacity:

Heat (million Btu/hr):

Steam : $25,000 \mathrm{lbs} / \mathrm{hr}$ (Randle)

40,000 lbs/hr (Morton) @300 psig

Electrical Generation (MW): None

Average Plant Output:

Heat (million Btu/hr):

Steam: 16,250 lbs/hr (Randle); 26,000 lbs/hr

(Morton) both @ 300 psig

Electrical Generation (MW): None

Type of Combuster/Furnace:

Manufacturer:

Type of Boiler: Water tube and fire tube

Boller Manufacturer: Seattle

Type of Pollution Control: Water scrubber

Reason for Using Wood Fuel: Economics

\section{Dalshowa America}

P.O. Box 271

Port Angeles, WA 98362

Phone: (206) 457-4474

Contact: T. Allen

Type of Business: Pulp and paperboard

Type of Biomass Fuel: Hog fuel

Source of Fuel: Purchased

Annual Biomass Fuel Consumed: 53,000 BDUs

Back-up Fuel: No. 6 fuel oil

End Use(s): Process steam, drying

Plant Design Capacity: 1 hog fuel boiler Heat (million Btu/hr):

Steam: 105,000 lbs/hr@255 psig

Electrical Generation (MW): None

Average Plant Dutput:

Heat (million Btu/hr):

Steam: 75,000 lbs/hr @ 255 psig

Electrical Generation (MW): None

Type of Combuster/Furnace: Spreader stoker

Manufacturer: Combustion Engineering

Type of Boile:: Model VU-40

Boiler Manufacturer: Combustion Engineering

Type of Pollution Control: Venturi Scrubber

Reason for Using Wood Fuel: Economics

\section{E. A. Nord Company}

300 W Marine Drive

Everett, WA 98201

Phone: (206) 259-9292, ext. 445

Contact: Ted Davis

Type of Business: Doors

Type of Biomass Fuel: Hog fuel

Source of Fuel: In-house

Annual Biomass Fuel Consumed: 10,000

tons/year

Back-up Fuel: None

End Use(s): Dry kiln and slicer tank

Plant Design Capacity:

Heat (million Btu/hr):

Steam: 55,000 lbs/hr@ psig

Electrical Generation $(\mathrm{MW})$ : None

Average Plant Output:

Heat (million Btu/hr):

Steam: 15,000 lbs/hr@ psig

Electrical Generation $(\mathrm{MW})$ :

Type of Combuster/Furnace:

Manufacturer:

Type of Boiler: Fire tube

Boiler Manufacturer: Wyatt Kipper

Type of Pollution Control: Multi-clone

Reason for Using Wood Fuel:

\section{Fort Vancouver Plywood}

P.O. Box 289

Vancouver WA 98660

Phone: (206) 694-3368

Contact: Duane Grover

Type of Business: Plywood

Type of Biomass Fuel: Hog fuel, sawdust

Source of Fuel: In-house

Annual Biomass Fuel Consumed: 35,000 units

Back-up Fuel: None

End Use(s): Process heat

Plant Design Capacity: Heat (million Btu/hr):

Steam: 75,000 lbs/hr@300 psig

Electrical Generation (MW): None

Average Plant Output:

Heat (million Btu/hr):

Steam: 45,000 lbs/hr @ 290 psig

Electrical Generation (MW): None 
Type of Combuster/Furnace: None

Manufacturer:

Type of Boiler: Hog fuel fired, water lube

Boiler Manufacturer: Kipper \& Sons

Type of Pollution Control: Wet scrubber

Reason for Using Wood Fuel: By-product of manufacturing

Fred Tehb \& Sons, Incorporated

1906 Mall Streot

Tacoma, WA 98421

Phone: (206) 272-4107

Contact: Charles Hoftman

Type of Business: Remanufacture of lumber products

Type of Blomass Fuel: Sawdust

Source of Fuel: In-house

Annual Biomass Fuel Consumed: 219 tons

Back-up Fuel: Natural gas, oil

End Use(s): Drying of lumber

Plant Design Capacity:

Heat (million Btu/hr):

Steam: 15,000 lbs/hr@10 psig

Electrical Generation (MW):

Average Plant Output:

Heat (million Btu/hr):

Steam: 15,000 lbs/hr@10 psig

Electrical Generation (MW):

Type of Combuster/Furnace: Oil, gas, wood

Manufacturer: Rayburn

Type of Boiler: Fire tube

Boiler Manufacturer: Cleaver

Type of Pollution Control: Baghouse

Reason for Using Wood Fuel: Economics

\section{Georgla-Paciflc Corporation}

P.O. Box 1236

Bellingham, WA 98225

Phone: (206) 733-4410

Contact: E. H. Dahlgren

Type of Business: Pulp and paper Type of Biomass Fuel: Hog fuel

Source of Fuel: Purchased \& generated

Annual Biomass Fuel Consumed: 157,000 bone dry tons

Back-up Fuel: Natural gas/No. 6 oil
End Use(s): Process steam, drying

Plant Design Capacity:

Heat (million Btu/hr): 232

Steam: $220,000 \mathrm{lbs} / \mathrm{hr} @ 200 \mathrm{psig}$

Electrical Generation (MW): None

Average Plant Output:

Heat (million Btu/hr): 185

Steam: 175,000 lbs/hr @ 175 psig

Electrical Generation (MW): None

Type of Combustion/Furnace: Dutch oven bollers

Manufacturer: Babcock \& Wilcox

Type of Boiler: Sterling

Boiler Manufacturer: Babcock \& Wilcox

Type of Pollution Control: Baghouse

Reason for Using Wood Fuel: Economics

\section{Goodyear Nelson Hardwood Lumber Company, Incorporated}

1215 Highway $9 \mathrm{~S}$

Sedro Woolley, WA 98284

Phone: (206) 856-0816

Contact: Rod Remingion

Type of Business: Hardwood lumber

Type of Biomass Fuel: Hog fuel and shavings

Source of Fuel: Western hardwood residue and surfacing

Annual Biomass Fuel Consumed:

Back-up Fuel:

End Use(s): Steam for dry kilns

Plant Design Capacity:

Heat (million Btu/hr):

Steam: 25,000 lbs/hr@110 psig

Electrical Generation (MW): N/A

Average Plant Output:

Heat (million Btu/hr):

Steam: 19,500 lbs/hr@110 psig

Electrical Generation (MW): None

Type of Combuster/Furnace:

Manufacturer: G\&S Retrofit Auto Mini-Stoker System

Type of Boiler: Fire tube

Boiler Manufacturer: Brownell

Type of Poliution Control: None

Reason for Using Wood Fuel: Availability 
Hardel Mutual Plywood

1515 West Bay Drive

Olympla, WA 98502

Phone: (206) 754-6030

Contact: Vic Durham

Type of Business: Plywood

Type of Blomass Fuel: Sander dust \& hog fuel

Source of Fuel: In-house

Annual Blomass Fuel Consumed:

Back-up Fuel: Natural gas

End Use(s): Dryers and presses

Plant Design Capacity:

Heat (million Btu/hr):

Steam: 28,000 lbs/hr @ 250 psig

Electrical Generation (MW):

Average Plant Output:

Heat (million Btu/hr)

Steam: 25,000 lbs/hr @

Electrical Generation (MW): psig

Type of Combuster/Furnace: Suspension burner

Manufacturer: Coen

Type of Boller: Fire tube

Boiler Manufacturer: Pacific Marion

Type of Pollution Control: Joy multicone

Reason for Using Wood Fuel:

\section{ITT Rayonler, Incorporated - Grays \\ Harbor DIvision}

23rd and Railroad Streets

Hoquiam, WA 98550

Phone: (206) 532-1410

Type of Business: Pulp

Type of Biomass Fuel: Hog fuel (wood refuse, bark)

Source of Fuel: Area sawmills and chip facilities

Annual Biomass Fuel Consumed: 200,000 bone dry metric tons

Back-up Fuel:

End Use(s):

Plant Design Capacity:

Heat (million Btu/hr):

Steam: $325,000 \mathrm{lbs} / \mathrm{hr}$ @ $350 \mathrm{psig}$

Electrical Generation (MW): 0
Average Plant Output:

Heat (million Btu/hr):

Steam: 300,000 lbs/hr@

Electrical Generation (MW): psig

Type of Combuster/Furnace: 2 Hog fuel boilers

Manufacturer: Flley and Bomstead Wolford

Type of Boller:

Boiler Manufacturer:

Type of Pollution Control: Fly ash multicones and wet scubbers

Reason for Using Wood Fuel:

\section{ITT Rayonler, Incorporated}

P.O. Box 191

Port Angeles, WA 98362

Phone: (206) 457-3391

Contact: D. E. Collings

Type of Business: Pulp

Type of Biomass Fuel: Hog fuel, primary \& secondary sludge mix

Source of Fuel: Purchased and produced

Annual Blomass Fuel Consumed: 84,000 bone dry metric tons

Back-up Fuel: Fuel oil

End Use(s): Process steam, dryers

Plant Design Capacity:

Heat (million Btu/hr): 290

Steam: 200,000 Ibs/hr@425 psig

Electrical Generation (MW): 12.0

Average Plant Output:

Heat (million Btu/hr): 254

Steam:__lbs/hr@425 psig

Electrical Generation (MW): 2.7

Type of Combuster/Furnace:

Manufacturer: Bumstead. Woolford

Type of Boiler: Pin hole stationary grate

Boiler Manufacturer: Riley

Type of Pollution Control: Barron Cinder Collectors and Combustion Power Gravel Scrubbers

Reason for Using Wood Fuel: By-product of pulp production 


\section{James Rlver}

NE Fourth \& Adams Street

Camas, WA 98607

Phone: (206) 834-8161

Contact: W. W. Vautzelfden

Type of Business: Pulp and paperboard

Type of Blomass Fuel: Hog fuel

Source of Fuel: Purchased

Annual Blomass Fuel Consumed: $20 \mathrm{MM}$ Btu/hr.

Back-up Fuel: Gas/oll

End Use(s): Process steam, dryers

Plant Design Capacity:

Heat (million Btu/hr): 138

Steam: $130,000 \mathrm{lbs} / \mathrm{hr} @ 400 \mathrm{psig}$

Electrical Generation (MW): 9

Average Plant Output:

Heat (million Btu/hr): 84

Steam: 80,000 lbs/hr@ 900 psig

Electrical Generation (MW): 7

Type of Combuster/Furnace: Dutch oven

Manufacturer: Puget Sound Machinery Depot (PSMD)

Type of Boller: PSMD

Boiler Manufacturer: PSMD

Type of Pollution Control: Centricleaner

Reason for Using Wood Fuel: Cost

\section{Jeld-Wen Fiber of Washington}

Corner of Wesley \& Fort Roads

White Swan, WA 98952

Phone: (509) 874-2255

Contact: Tom Wilson

Type of Business: Fiberboard doorskins

Type of Biomass Fuel: Wood

Source of Fuel: Plant waste

Annual Biomass Fuel Consumed: 10,000 15,000 tons

Back-up Fuel:

End Use(s): Plant space heat, process heat

Plant Design Capacity:

Heat (million Btu/hr):

Steam:30,000 lbs/t:r@200 psig

Electrical Generation (MW): None
Average Plant Output:

Heat (million Btu/hr):

Sleam: 13,000 - 17,000 lbs/hr (iv $200 \mathrm{psig}$

Electrical Generation (MW): None

Type of Combuster/Furnace: 2 cell forced draft

Manufacturer: Wellons

Type of Boller: Water tube

Boller Manufacturer: Nebraska

Type of Pollution Control: Mechanical, multicone scrubber

Reason for Using Wood Fuel: Availability of wood waste

\section{KInnear of Washlngton}

2001 Industrial Drive

Centralia, WA 98531

Phone: (206) 736-7651

Contact: Greg Penley

Type of Business: Doors, mouldings

Type of Blomass Fuel: Mill residues

Source of Fuel: In-house

Annual Biomass Fuel Consumed: 1,100 tons

Back-up Fuel: None

End Use(s): Drying lumber

Plant Design Capacity:

Heat (million Btu/hr): Unknown

Steam: 10,300 lbs/hr@100 psig

Electrical Generation (MW): None

Average Plant Output:

Heat (million Btu/hr): Unknown

Steam: 8,250 Ibs/hr@80 psig

Electrical Generation (MW): None

Type of Combuster/Furnace: Firebox

Manufacturer: Frye Shaving Feeder

Type of Boiler: Fire tube

Boiler Manufacturer: Kewanee \#590

Type of Pollution Control: Wet scrubber

Reason for Using Wood Fuel: Avallable/low cost 
K-Ply Inc.

439 Marine Drive

Port Angeles, WA 98362

Phone: (206) 457-4421

Contact: John Doane

Type of Business: Plywood

Type of Blomass Fuel: In-house

Source of Fuel: Bark, veneer trim, plywood trim

Annual Biomass Fuel Consumed: 24,000 BDTs (estimate)

Back-up Fuel: None

End Use(s): Drying, veneer

Plant Design Capacity:

Heat (million Biu/hr):

Steam: 50,000lbs/hr@150 psig (2 bollers 25,000 hr ea.)

Electrical Generation (MW): None

Average Plant Output:

Heat (million Btu/hr):

Steam: 40,000 lbs/hr@140 psig

Electrical Generation (MW): None

Type of Combuster/Furnace:

Manufacturer: Riley

Type of Boiler: "RO"

Boller Manufacturer: Riley

Type of Pollution Control: Wet E.S.P.

Reason for Using Wood Fuel: Avallability

\section{Layman's Lumber Company}

15930 Highway 12

Naches, WA 98937

Phone: (509) 653-2221

Contact: George Layman

Type of Business: Lumber

Type of Blomass Fuel: Hog fuel

Source of Fuel: In-house

Annual Biomass Fuel Consumed: 3,000 tons

Back-up Fuel:

End Use(s): Dry kilns

Plant Design Capacity:

Heat (million Btu/hr):

Stearn: 17,250 lbs/hr@15 psig

Electrical Generation (MW):
Average Plant Output:

Heat (million Btu/hr):

Steam: 15,000 lbs/hr @15 psig

Electrical Generation (MW):

Type of Combuster/Furnace:

Manufacturer: Wellons

Type of Boiler: Fire tube

Boiler Manufacturer:

Type of Pollution Control: Recycle

Reason for Using Wood Fuel:

\section{Longulew Fibre Company}

P.O. BOX 3000

Longview, WA 98632

Phone: (206) 425-1550

Contact: R. W. Williams

Type of Business: Pulp and paperboard

Type of Blomass Fuel: Hog fuel

Source of Fuel: Purchased

Annual Biomass Fuel Consumed: 232,812 dry tons

Back-up Fuel: Fuel oll, natural gas

End Use(s): Process steam, dryers

Plant Design Capacity: 3 hog fuel boilers Heat (million Btu/hr): 1,160

Steam: $850,000 \mathrm{lbs} / \mathrm{hr} @ 800$ psig (2 boilers

@275,000, 1 boller@300,000)

Electrical Generation (MW): 30 (Hog fuel portion of total)

Average Plant Output: Calendar 1989 (hog fuel $38 \%$ fuel used in above boilers)

Heat (million Btu/hr): 265 (hog fuel portion)

696 total

Steam: 196,296 lbs/hr @ 800 psig (hog fuel portion)

Electrical Generation (MW): 23 (hog fuel portion)

Type of Combuster/Furnace: N/A

Manufacturer: N/A

Type of Boiler: 2 Dutch ovens, 1 StokerTraveling Grate

Boller Manufacturer: 2 Babcock \& Wilson, 1 Combustion Engineering

Type of Pollution Control: Multicone \& wet scrubbers

Reason for Using Wood Fuel: Lowest cost fuel 


\section{Loulslana Paclfic Company}

P.O. Box 1575

Walla Walla, WA 98362

Phone: (509) 52.9-02.00

Contact:

Type of Business: Lumber

Type of Blomass Fuel: Hog fuel

Source of Fuel: In-house

Annual Blomaš̃ Fuel Consumed: 12,000 tons

Back-up Fuel: 0

End Use(s): Dry kilns

Plant Design Capacity: Heat (million Btu/hr): $21,000,000$

Steam: 20 to 25,000 lbs/hr @ Electrical Generation (MW): None

Average Plant Output:

Heat (million Btu/hr): $14,060,000$

Steam: 13,400 Ibs/hr @___. psig

Electrical Generation $(\mathrm{MW})$ : None

Type of Combuster/Furnace:

Manıufacturer: Seattle Boiler Works

Type of Boller: Dutch oven 84-20 hrt 125 lbs.

Boller Manufacturer: Seattle Boiler Works

Type of Pollution Control: Proper fuel \& air ratio

Reason for Using Wood Fuel: Cost

\section{Mt. Baker Plywood}

P.O. Box 997

Bellingham, WA 98225

Phone: (206) 733-3960

Contact: Harley Willis

Type of Business: Plywood

Type of Biomass Fuel: Hog fuel

Source of Fuel: In-house

Annual Blomass Fuel Consumed:

Back-up Fuel: Natural gas

End Use(s): Drying and pressing

Plant Design Capacity:

Heat (million Btu/hr:

Steam: 35,000 lbs/hr@_..psig

Electrical Generation (MW): None

Average Plant Output:

Heat (million Btu/hr):

Steam: 31,500 lbs/hr@

Electrical Generation $(\mathrm{MW})$ :
Type of Combuster/Furnace:

Manufacturer:

Type of Boller:

Boller Manufacturer:

Type of Pollution Control:

Reason for Using Wood Fuel:

\section{Northwest Hardwoods}

20015 - 67th NE

Arlington, WA 98223

Phone: (206) 435-8502

Contact: Roy Popper

Type of Business: Lumber

Type of Blomass Fuel: Sander dust

Source of Fuel: In-house

Annual Blomass Fuel Consumed: Unknown

Back-up Fuel: Natural gas

End Use(s): Dry kilns

Plant Design Capacity:

Hea: (million Btu/hr):

Steam: 18,000 lbs/hr@22 psig

Electrical Generation (MW):

Average Plant Output:

Heat (million Btu/hr):

Stearn: 18,000 lbs/hr @22 psig

Electrical Generation (MW):

Type of Combuster/Furnace:

Manufacturer:

Type of Boller: Fire tube

Boiler Manufacturer: Cleaver Brooks

Type of Pollution Control: 3 cell bag hause

Reason for Using Wood Fuel: It is produced

in our lumber finishing process

\section{Oeser Company, The}

730 Marine Drive

Bellingham, WA 98225

Phone: (206) 733-5184

Contact: Ronald S. Durbin, Plant Manager

Type of Business: Wood treating

Type of Blomass Fuel: Hog fuel

Source of Fuel: In-house

Annual Biomass Fuel Consumed: 400 tons (est.)

Back-up Fuel: Natural gas 
End Use(s): Steam heat for treating prooesses

Plant Design Capacity:

Heat (million Btu/hr): 250 - 400 bhp

Steam: Ibs/hr @ 120 psig

Electrical Generation (MW): None

Average Plant Output:

Heat (million Btu/hr): 150 bhp

Steam: Ibs/hr@120psig

Electrical Generation (MW): None

Type of Combuster Furnace: Dutch oven

Manufacturer: Constructed on sight by plant personnel

Type of Boller: Water tube

Boller Manufacturer: Sterling

Type of Pollution Control: Visual at stack/mix with natural gas

Reason for Using Wood Fuel: Because it is a manufacturing by-product, economical, a rellable supply, and otherwise wasted if not used for fuel

\section{Omak Wood Products, Incorporated \\ Route 2, Box 54 \\ Omak, WA 98841 \\ Phorie: (509) 826-1460, ext. 238 \\ Contact: W. C. Dufton, P.E., Engineering Manager}

Type of Business: Lumber and plywood Type of Blomass Fuel: Hog fuel, sawdust Source of Fuel: In-house

Annual Blomass Fuel Consumed: 175,200 units

Back-up Fuel: Natural gas

End Use(s): Dry kilns and veneer presses, power generation

Plant Design Capacity:

Heat (million Btu/hr):

Steam: 240,000 lbs/hr @675 psig)

Electrical Generation (MW): 7.5

Average Plant Output:

Heat (million Btu/hr):

Steam: 160,000 lbs/hr @ 600 psig

Electrical Generation (MW): 7.5

Type of Combuster/Furnace: 2 air suspension type burners

Manufacturer: Riley Boller

Type of Boller: Water tube
Boller Manufacturer: Rlley Stoker, 2 units

Type of Pollution Control: Wel sorubber

Reason for Using Wood Fuel: By-product of production, disposes production residues

\section{Paclflc Wood Treating}

111 W Division

Ridgefield, WA 98642

Phone: (206) 887-3562

Contact: Al Dwyer

Type of Business: Treated lumber \& re manufacturing In wood products

Type of Blomass Fuel: Hog fuel

Source of Fuel: In-house and other wood fabrication industries

Annual Blomass Fuel Consumed: 12,500 units

Back-up Fuel: Diesel and natural gas

End Use(s): Process heat

Plant Design Capacity:

Heat (million Btu/hr):

Steam: 40,000 Ibs/hr @___._psig

Electrical Generation ( $\mathrm{MW}$ ): None

Average Plant Output:

Heat (million Btu hr):

Steam: 20,000 lbs/hr@ @ psig

Electrlcal Generation (MW): None

Type of Combuster/Furnace:

Manufacturer: Wellons

Type of Boiler: Trame water tube

Boller Manufacturer: Trame

Type of Pollution Control: Baghouse

Reason for Using Wood Fuel: Price

\section{Plum Creek Manufacturing, Incorporated}

Arden Lumber Operations

634 Highway $395 \mathrm{~S}$

Colville, WA 99114

Phone: (509) 684-5084

Contact: John Chopot

Type of Business: Lumber

Type of Biomass Fuel: Hog fuel

Source of Fuel: In-house, sawmill

Annual Blomass Fuel Consumed:

Back-up Fuel: None 
End Use(s): Dry kllns

Plant Design Capaclty:

Heat (million Btu/hrr):

Steam: lbs/hr@150 psig

Electrical Generatlon (MW): None

Average Plant Output:

Heat (million Btu/hr):

Steam: $\quad$ bs/hr @

Eleotrical Generation (MW): None

Type of Combustion/Furnace:

Manufacturer: Klpper \& Son

Type of Boller: Water tube

Boller Manufacturer: Kipper \& Son

Type of Pollution Control: Wet scrubber

Reason for Using Wood Fuel: Avallability cost

\section{Port Townsend Paper}

P.O. Box 3170

Port Townsend, WA 98368

Phone: (206) 385-3170

Contact: [lick D'Agostino

Type of Business: Pulp and paper

Type of Blomass Fuel: Hog fuel

Source of Fuel: Purchased

Annual Blomass Fuel Consumed: $77,713 \mathrm{dry}$ tons

Back-up Fuel: Residual oil

End Use(s): Process steam and dryers

Plant Design Capacity: 2 hog fuel

Heat (million Btu/hr):

Stearn: 250,000lbs/hr@600psig

Electrical Generation (MW): 18.5

Average Plant Output:

Heal (million Btu/hr):

Steam: 133,000 lbs/hr@ @ 400 psig

Electrical Generation (MW): 6.1

Type of Combuster/Furnace:

Manufacturer: Combustion Engineering

Type of Boller: VU 40

Boller Manufacturer:

Type of Pollution Control: Mechanical collectors plus wet scrubber

Reason for Using Wood Fuel: Cost

\section{Puget Sound Plywood}

230 F Street

Tacoma, WA 98421

Phone: (206) 627-4111

Contaot:

Type of Business: Plywood

Type of Blomass Fuel: Sawdust, mill residues

Source of Fuel: In-house \& purchased

Annual Blomass Fuel Consurned: 17,000 tons

Back-up Fuel: Natural gas

End Use(s): Dryers

Plant Design Capacily:

Heat (million Btu/hr):

Steam:500hp@_.psig

Electrical Generation (MW):

Average Plant Output:

Heat (milllon But/hr): 35

Steam: Ibs/hr @ 180 psig

Electrical Generation (MW):

Type of Combuster/Furnace: Dutch oven

Manufacturer: Sterling

Type of Boller: Water tube

Boller Manufacturer: Sterling

Type of Pollution Control: Joy Multicone

Reason for Using Wood Fuel: Economics

\section{San Poll Lumber Company}

P.O. Box 3577

Republic, WA 99166

Phone: (509)

Contact:

Type of Business: Lumber

Type of Blomass Fuel: Hog fuel

Source of Fuel: In-hause

Annual Blomass Fuel Consumed:

Back-up Fuel:

End Use(s): Dry klln

Plant Design Capacity:

Heat (million Btu/hr):

Steam: 50,000 lbs/hr @

Electrical Generation (MW): psig

Average Plant Output:

Heat (million Btu/hr):

Steam: 30,000 lbs/hr@

Electrical Generation (MW): psig 
Type of Combuster/Furnace: 1D-FD

Manufaoturer: Wellons

Type of Boller: Woodwaste flred (bark, etc.)

Boller Manulacturer: Nobraska

Type of Pollution Control: $8-10 \mathrm{CO}_{2}$

Reason for Using Wood Fuel: On plant wood waste

\section{Scott Paper}

2600 Federal Avenue

Everett, WA 98220

Phone: (206) 259-7333

Contad: Alex M. Hood

Type of Business: Pulp and paper

Type of Blomass Fuel: Hog fuel

Source of Fuel: Purchased and In house

Annual Blomass Fuel Consumed: 140,000 units

Baok-up Fuel: \#6 oll

End Use(s): Process steam, drying

Plant Design Capacity: 11 bollers of which 5 are hog fuel

Heal (milllon Btu/hr):

Steam: $5 @ 50,000 \mathrm{lbs} / \mathrm{hr} @$ psig (hog

fuel), $1 @ 120,000 \mathrm{lbs} / \mathrm{hr}, 3$ @ 150,000 lbs//hr,

1 @ 380,000 lbs/hr

Electrical Generation (MW): None

Average Plant Output:

Heal (million Btu/hr):

Steam: $650,000 \mathrm{lbs} / \mathrm{hr} @ 22.5 \& 300 \mathrm{psig}$

Electrical Generation (MW): None

Type of Combuster/Furnace: Dutch oven

Manufacturer: Puget Sound Machinery

Depot, B\&W Modiffcation

Type of Boller: 4 drum Stirling

Boller Manufacturer: Same as above

Type of Pollution Control: Baghouse

Reason for Using Wood Fuel: Economles

\section{Simpson Door Company}

McCleary Operations

McCleary, WA 98557

Phone: (206) 495-3291

Contact: Robert K. Horton

Type of Business: Doors

Type of Blomass Fuel: Hog fuel

Source of Fuel: In-house
Annual Blomass Fuel Ccnsumed: 20,400 units

Back-up Fuel: Purchased hog fuel

End Use(s): Steam for wood drying and heatlig

Plant Dosign Capacliy:

Heat (million Btu/hr): N/A

Steam 45,000 lbs/hr @ 250 psig:

Electrloal Generatlon (MW): None

Average Plant Output:

Heat (million Btu/hr): N $N / A$

Steam: 25,000 lbs/lir @ 150 pslg

Electrical Generation (MW): None

Type of Combuster/Furnace: Dutoh oven

Manufacturer: Batomhauser

Type of Boller: Water lube - 3 pass

Boller Manufacturer: Baterihauser

Type of Pollution Control: All dry fuel .

Baghouses on fuel handling

Reason for Using Wood Fuel: By product of manufacturing doors

\section{SImpson Tacoma Kraft Company}

801 Portland Avenue

Tacoma, WA 98421

Phone: (206) $572-2150$ or $596-0257$

Contact: Davld McEntee

Type of Business: Pulp and paper

Type of Blomass Fuel: Hog fuel

Source of Fuel: Purchased

Annual Blomass Fuel Consumed: 182,000

Units (approx. equal to 174,000 BDT)

Back-up Fuel: Bunker C

End Use(s): Process steam, dryers

Plant Design Capacity: 1 hog fuel

Heat (million Btu/hr): 254

Sleam: $225,000 \mathrm{lbs} / \mathrm{hr} @ 425 \mathrm{psig}$

Electrical Generation (MW): None

Average Plant Output:

Heat (million Btu/hr): 231,000

Steam: 205,000 lbs/hr (iv $425 \mathrm{psig}$

Electrical Generation (MW): None

Type of Combuster/Furnace: Dis-

tributor/Detrolt

Hyord-Grate

Manufacturer: Detrolt, Stoker

Type of Boller: 2 drum natural olrculation

Boiler Manufacturer: Riley 
Type of Pollutlon Control: Electro Statlc Preclpltator

Heason for Using Wood Fuol: Eoonomlos

\section{Simpson TImber Company \\ Waterfront Streot \\ Shelton, WA 98584 \\ Phone: (206) 426-3381 \\ Contad: Charlle Swenson}

Type of Business: Lumber and plywood Type of Blomass Fuel: Hog fuel

Source of Fuel: In-house

Annual Blomass Fuel Consumed:

Back-up Fuel: None

End Use(s): Dryling, lumber and plywood veneers

Plant Dealgn ciapaolty: 6 fuel colls Heat (million Btu/hr): 140, approx. Steam: $140,000 \mathrm{lbs} / \mathrm{hr}$ @ $300 \mathrm{psig}$ Electrical Generation (MW): None

Average Plant Output: Heat (million Btu/hr): 130 approx. Steam: $130,000 \mathrm{lbs} / \mathrm{hr} @ 300 \mathrm{psig}$ Electrical Generation (MW): None

Type of Combuster/Furnace: Fuel oell

Manufacturer: Welloris

Type of Boller: Water tube

Boller Manulacturer: Wellons

Type of Pollution Control: Two stage combus. tlon plus multi-oones

Heason for Using Wood Fuel: Lowest cost, In. house fuel

\section{Smith Street MIII, Incorporated \\ 3600 Smlth Street \\ Everett, WA 98201 \\ Phone: (206) 252-7179 \\ Contaol: Bob Buse}

Type of Business: Alcler sawmlll and planning mill

Type of Blomass Fuel: Alder sawdust and dry shavings

Source of Fuel: Sawmill

Annual Blomass Fuel Consumed:

Back-up Fuel: None

End Use(s): Heat dry killns
Plant Deslgn Capaolty:

Heat (million Btu/hr):

Steam:_ Ibs/hr (ci) $15 \mathrm{psig}$

Eleotrical Generatlon (MW): None

Average Plant Output:

Heat (million Btu/hr):

Stealln: Ibs/hr @ $15 \mathrm{ps} / \mathrm{g}$

Eleotrical Generation (MW): None

Type of Combuster/Furnace: Dutoh oven

Manufaoturer: In-house

Type of Boller: Low pressure, $15 \mathrm{lbs}$.

Boller Manufacturer: Brownell

Type of Pollutlon Control:

Reason for Using Wood Fuel: Economles

\section{Stevenson Co-Ply, Incorporated}

P.O. BoxF

Stevenson, WA 98648

Phone: (509) 42\%-5020

Contact: Pat Swoeney

Type of Business: Plywood

Type of Blomass Fuel: Hog fuel

Source of Fuel: In-house

Annual Blomass Fuel Consumed: 11,350 units

Back-up Fuel: None

End Use(s): Drying

Plant Design Capacity:

Heal (million Btu/hr): None

Steam: 40,000 lbs/hr @ 250 psig

Electrical Generation (MW): Norie

Average Plant Output:

Heat (milllon Btu/hr): None

Steam: 35,000 lbs/hr @ 250 psig

Electrical Generation (MW): None

Type of Combuster/Furnace:

Manufaoturer:

Type of Buller: Dutch oven

Boller Manufacturer: Wyatt \& Klpper

Type of Pollution Control:

Reason for Using Wood Fuel: Availabllity, cost 
Strauser Marlufacturing Company 2921 Melrose Street

Walla Walla, Washington 99362

Phone: (509) 529-6284

Contaot: Beverly Strauser

Type of Business: Lincoln Logs

Type of Blomass Fuel: Hogfuel, sawdust, shavings

Back-up Fuel:

Source of Fuel: In-house

Anrual Biomass Fuel Consumed: 3000 dry tons

End Use(s): Space and process heat

Plant Deslgn Capaoily: Heat (million Btu/hr:

Steam: 6,000 lbs/hr @ 250 pslg

Electrical Generation (MW):

Average Plant Output:

Heat (million Btu/hr):

Steam: 4,800 Ibs@ psig

Eleotrioal Generatlon (MW):

Type of Combuster/Furnace: Suspension Gaslfier

Manufaoturer:

Type of Boller: $250 \mathrm{hp}$ Cleaver Brooks

Manufaoturer:

Type of Pollution Control: Flash collector

Reason for Use : Economios

\section{Summit TImber Power House \\ 29917 Bennittville Store Road \\ Darrington, WA 98241 \\ Phone: (206) 259-4185, ext. 121 \\ Contact: Ed McDaniel, Power House Superintendent}

Type of Business: Wood Products

Type of Blomass Fuel: Hog fuel

Source of Fuel: In-house

Annual Blomass Fuel Consumed: 36,500 tons

Back-up Fuel: Bunker C Fossll (oll)

End Use(s):

Plant Design Capacity:

Heat (million Btu/hr):

Steam: 45,000 lbs/hr@150 psig

Electrical Generation (MW): None
Average Planl Output:

Heat (milllon Btu/hr):

Steam: $1 \mathrm{bs} / \mathrm{hr}$ (c) psig

Eleotrical Ceneratlon (MWV): None

Type of Combuster/Furnace:

Manufaoturer: Puget Sound Boller

Type of Boller: Water tube

Boller Manufaoture:

Type of Pollution Control: Breslove Separator

Reason for Using Wood Fuel: Cut fuel cosib, Inoinerate wood waste

\section{Vaagen Brothers Lumber, Incorporated}

565 W Fitth

Colville, WA 99114

Phone: (509) 684.5071

Contaot: Jim Winninges, Supt.

Type of Business: Lumber

Type of Blomass Fuel: Hog fuel

Source of Fuel: innouse

Annual Blomass Fuel Consumed: 25,550

tons

Back-up Fuel: No

End Use(s): Dry kilns, generator

Plant Design Capacity:

Heat (million Btu/hr):

Stearm: 60,000 lbs/tir @ $510 \mathrm{psig}$

Electrical Generatlon (MW): $4,000 \mathrm{kWh}$

Average Plant Output:

Heal (million Btu/hr):

Steam: 60,000 lbs/hr @ $510 \mathrm{psig}$

Electrical Generation (MW): 4,000 kW

Type of Combuster/Furnace: Fuel cell 4 oells

Manufacturer: Wellons

Type of Boller: Water tube

Boller Manufacturer: Nebraska

Type of Pollutlon Control: Multiclones

Reason for Using Wood Fuel: Economles 
Vaagen Brothers Lumber, Incorporated P.O. Box 257

Ione, WA 99139

Phone: (609) 442.3511

Contact: Don Curry, Plant Manager

Type of Buslriess: Lumber

Type of Blomass Fuel: Hog Fuel

Source of Fuel: In-house

Annual Blomass Fuel Consumed: 9,490 tor,s

Back.up Fuel:

End Use(s): Dry kilns

Plant Design Capaclty:

Heat (million Bfu/hr): 12.5

Stearn: 40,000 lbs/hr @ 150 psig

Eleotrloal Generation (MW):

Average Plant Output:

Heat (million Btu/hr):

Steam: $30,000 \mathrm{lbs} / \mathrm{hr} @ 150 \mathrm{psig}$

Electrical Generation MW):

Type of Combuster/Furnace: Radiant furnace healing surface

Manufacturer: Nebraska Boller Company

Type of Boller: Water tube

Boller Manufacturer: Nebraska Boller Company

Type of Pollution Control: Cleveland Controls

Feason for Using Wood Fuel: Cost efflclent

West Coast Forest Products, incorporated

19406 68th Drlve NE

P.O. B0. 365

Arlington, WA 98223

Phone: (206) 435-2175

Contaot: Jim Reece

Type of Business: Lumber remanufacturer

Type of Blomass Fuel: Shavings

Source of Fuel: Planer

Annual Blomass Fuel Consumed: 4,7'20 dry Ions

Back-up Fuel: None

End Use(s): Dry kilns

Plani Design Capacity:

Heat (million Btu/hr): 22.5

Steam:__lbs/hr@ psig

Elecirical Gerieration (MW): N/A
Averauril lant Output:

Heat (m)lllon Btu/hr):

Steam: Ibs/hr@100 psig

Electrical Generation (MW): None

Type of Combuster/Furnaoe:

Manulacturor:

Type of Boller: F're tube

Boller Manufacturer: Titusville

Type of Pollution Control: Mlkropul baghouse

Feason for Using Wood Fuel: Cost and avallabllity

\section{Weyerhaeuser Company}

P.O. Box 1000

Cosmopolis, WA 98537

Phone: (206) 532-7110

Contaot: Paul W. Kelso, P\&R Superintendent

Type of Business: Pulp

Type of Blomass Fuel: Hog fuel

Source of Fuel: Purchased \& In house generated

Annual Blomass Fuel Consumed: 80,000 BD'T

Back-up Fuel: No. 6 fuel oll

End Use(s): Process sloam

Plant Design Capaoity: Heat (million Btu/hr): 220

Steam: 160,000 lbs/hr@850psig

Electrical Generation (MW): 15.0

Average Plant Output: Heat (million Btu/hr): 138

Steam: 100,000 lbs/hr @850psig

Electrical Generation (MW): 11

Type of Combuster/Furnace: Fixed, water cooled, pin hole grate

Manufacturer: Babcox \& Wilcox

Type of Boller: Two Drum Sterling

Boller Manufacturer: Babcox \& Wilcox

Type of Pollution Control: Venturi wet scrubber

\& multiones

Reason for Using Wood Fuel: By-product of company-owned facilities 
Weyerhaouser Company

P.O. Box 188

Longview, WA 98632

Phone: (206) 425.2150

Contaot: Bob Lusk

Type of Business: Pulp and paperboard

Type of Blomass Fuel: Hog fuel

Source of Fuel: Purohased and in-house

Annual Blomass Fuel Consumed: 500,000 BDT

Back-up Fuel: Coal, oll, natural gas

End Use(s): Process steam, dryers

Plant Design Capacity: 7 hog fuel

Heat (million Btu/hr): 1,180

Steam: 550,000 lbs/hr@1,250 psl \& 300,000

lbs/hr@300 psl

Electrical Generation (MW): 30 (hog fuel portion of total)

Average Plant Output:

Heat (million Btu/hr): 1,000

Steam: 500,000 lbs/hr @ 1,250 psi \& 200,000

Ibs/hr @300 psi

Electrical Generation (MW): 30

Type of Combuster/Furnace:

Manufacturer: 6 Erie Clty, 1 Foster Wheeler

Type of Boller: Erle Clty Flxed Grate, Foster Wheeler Traveling Grate

Boller Manufacturer:

Type of Pollution Control: Wet Scrubber, Electrostatic Precipitator

Reason for Using Wood Fuel: By-product of company mills

\section{Weyerhaeuser Company}

P.O. Box 666

Raymond, WA 98577

Phone: (206) 942-2442

Contact: Jerry Dillayou, Plant Engineer

Type of Business: Lumber

Type of Blomass Fuel: Hog fuel

Source of Fuel: In-house

Annual Blomass Fuel Consumed:

Back-up Fuel: None - propane used to preheat on cold start-up

End Use(s): Dry kilns

Plant Design Capacity: Heat (million Btu/hr): $70,000,000$ (reduced to $55 \mathrm{MM}$ Btu/hr)
Steam: 40,000 lbs/hr@150psig

Electrical Generation (MW): None

Average Plant Output:

Heat (million Btu/hr): 48,000,000 est.

Steam: 37,000 lbs/hr @ psig

Electrical Generation (MW): None

Type of Combuster/Furnace:

Manufacturer: EPI $18 \mathrm{ft}$. dla. fluld bed burner (reduced to $16 \mathrm{H}$. dla.) built in 1974

Type of Boller: Combustion Engineeririg water tube boller

Boller Manufacturer:

Type of Pollution Control: Zurn multicone

Reason for Using Wood Fuel: Cost residual of lumber plant operation

\section{Weyerhaeuser Company}

7001 - 396th Street SE

Snoqualmie Falls, WA 98065

Phone: (206) 888-2511

Contact: Leon Clark, Facillty Supervisor

Type of Business: Lumber

Type of Blomass Fuel: Hog fuel

Source ol Fuel: In-house

Annual Biomass Fuel Consumed:

Back-up Fuel: Residual oil

End Use(s): Dry kilns

Plant Design Capacity: 2 bollers, 270,000 $\mathrm{ppH}$. saturated steam total

Heat (million Btu/hr):

Steam: 270,000 lbs/hr@_psig

Electrical Generation (MW): None

Average Dlant Output:

Heat (million Btu/hr): 247,800 Btu both

bollers

Steam: 128,000 lbs/hr @__.psig (both

bollers included)

Electrical Generation (MW): None

Type of Combuster/Furnace: Dutch oven and spreader stocker

Manufacturer: One 1969 F.E. Riley 250 lbs sat. steam 150,000 ppH, one $1930 \mathrm{~F}$.E. PSMD-155 lb sat. steam 120,000 ppH.

Type of Boiler: Water tube

Boller Manufacturer: Rilley 
Type of Pollution Control: Combustion Power Co., Inc. Model DS-800 dry scrubber

Reason for Using Wood Fuel: By-product

\section{W.I. Forest Products}

2302 E Mallon

Spokane, WA 99202

Phone: (509) 534-1561

Contact:

Type of Business: Lumber

Type of Biomass Fuel: Hog fuel

Source of Ful: In-house

Annual Biomass Fuel Consumed: Unknown

Back-up Fuel: Natural Gas

End Use(s): Dry kilns

Plant Design Capacity:

Heat (million Btu/hr):

Steam: 30,000 lbs/hr @ 125 psig

Electrical Generation (MW): None

Average Plant Output:

Heat (million Btu/hr):

Steam: 30,000 lbs/hr@125 psig

Electrical Generation (MW): None

Type of Combuster/Furnace:

Manufacturer: Combustion Engineering

Type of Boiler: Sterling 3-drum water tube

Boiler Manufacturer: Combustion Engineering

Type of Pollution Coritrol: Mikro-Pur Baghouse

Reason for Using Wood Fuel: Inexpensive, readily available

\section{Other Facilities}

\section{DeGoede Bulb Farms}

409 Mossyrock Proad W

Mossyrock, WA 98564

Phone: (206) 983-3773

Contact: Bob DeGoede

Type of Business: Greenhouse and nursery

Type of Biomass Fuel: Mill residue

Source of Fuel: Purchased

Annual Biomass Fuel Consumed: Approximately 65 truck loads (45' vans)

Back-up Fuel: Propane forced air unit heaters

End Use(s): Greenhouse heating

Plant Design Capacity:

Heat (million Btu/hr): 4,506,000 (depending on fuel moisture content)

Steam: lbs/hr@ $\operatorname{psig} N / A$

Electrical Generation (MW): N/A

Average Planit Output:

Heat (million Btu/hr): 3,000,000 Btu/hr.

Steam: Ibs/hr@ psig N/A

Electrical Generation (MW): N/A

Type of Combuster/Furnace: Bio-Combuster (gasificavion process)

Manufacturer: Bio-Mass Burner

Type of Boiler: Hot water $-210^{\circ} \mathrm{F}$

Boiler Manufacturer: Vaagen Timber Products

Type of Pollution Control: Gasificaion/cyclone

Reason for Using Wood Fuel: Least cost

\section{Maple Lane School}

20311 Old Highway 99 SW

Centralia, WA 98537

Phone: (206) 736-1361

Contact: Lonnie Bauman

Type of Business: School

Type of Biomass Fuel: Wood pellets

Source of Fuel: Purchased, Northwest Pellet Mills, Manke Fuel Lignetics

Annual Biomass Fuel Consumed: 2,043 tons Back-up Fuel: Oil, coal, natural gas

End Use(s): Space heat domestic hot water 
Plant Design Capacity: 2 bollers at $250 \mathrm{HP}$ each

Heat (million Btu/hr): $17,500,000$

Stealn: 20,000 Ibs/hr @ 100 psig

Electrical Generation (MW): None

Average Plant Output:

Heat (million Btu/hr): 4,197,945

Steam: 16,300 lbs/hr @ 90 psig (winter 12,000 summer)

Electrical Generation (MW): None

Type of Combuster/Furnace: Underfed stoker

Manufacturer: Combustion Service and Equipment

Type of Boiler: Fire tube

Boiler Manufacturer: Combustion Service and Equipment

Type of Pollution Control: Baghouse

Reason for Using Wood Fuel: Pollution control (air \& ash disposal), labor \& maintenance costs on fuel conveyor systems

\section{Washington Soldlers Home \& Colony}

P.O. Box 500

Orting, WA 98360

Phone: (206) 593-2045

Contact: John Buffington

Type of Business: Service

Type of Biomass Fuel: Wood pellets

Source of Fuel: Purchased

Annual Biomass Fuel Consumed: 1,930 tons (back-up fuel)

Back-up Fuel: Biomass (natural gas is the primary fuel)

End Use(s): Space heat and hot water

Plant Design Capacity:

Heat (million Btu/hr):

Steam: 24,500 lbs/hr @ 100 psig

Electrical Generation (MW): None

Average Plant Output:

Heat (million Btu/hr:

Steam: 3,000 Ibs/hr @__ p p psig

Electrical Generation (MW): None

Type of Combuster/Furnace: SpreaderStoker
Manufacturer: Wyatt \& Kipper and ONB TriFuel

Type of Boiler: Water tube and fire tube

Boiler Manufacturer:

Type of Pollution Control: Cyclone

Reason for Using Wood Fuel:

\section{Washington State PenItentlary}

P.O. Box 520

Walla Walla, WA 99362

Phone: (509) 525-3610

Contact: Jim Blodgett

Type of Business: State Penitentiary

Type of Biomass Fuel: Wood Pellets

Source of Fuel: Purchased, Lignetics of Idaho

Annual Blomass Fuel Consumed: 3,000 dry tons

Back-up Fuel: Natural gas

End Use(s): Space heat, laundry, kitchen

Plant Design Capacity: 3 boilers

Heat (million Btu/hr):

Steam: 75,000 Ibs/hr@125 psig

Electrical Generation (MW): None

Average Plant Output:

Heat (million Btu/hr)

Steam: 32,000 Ibs/hr@125 psig October -

April; 10,000 lbs/hr May - September

Electrical Generation (MW): None

Type of Combuster/Furnace: Stoker

Manufacturer: Wyatt \& Kipper

Type of Boiler: Water wall

Boiler Manufacturer:

Type of Pollution Control: Multicones

Reason for Using Wood Fuel: Emissions problems with coal

Washington Water Power Company

Kettle Falls, WA 99141

Phone: (509) 738-2449

Contact:

Type of Business: Electric Utility

Type of Biomass Fuel: Hog fuel

Source of Fuel: Purchased mill residue

Annual Biomass Fuel Consumed: 500,000 green tons 
Back-up Fuel: Natural gas

End Use(s): Electrical generation

Plant Design Capacity: Heat (million Btu/hr): N/A

Steam: 415,000 lbs/hr @ 1,500 psig

Electrical Generation (MW): 48 gross

Average Plant Output:

Heat (million Btu/hr): N/A

Steam: 435,000 lbs/hr@1,525 psig

Electrical Generation (MW): 50.3

Type of Combuster/Furnace: Spreader stoker, moving grate

Manufacturer: Combustion Engineering

Type of Boiler: Water tube

Boiler Manufacturer: Combustion Engineering

Type of Pollution Control: ESP and mechanical collector

Reason for Using Wood Fuel: Availability

\section{Western State Hospltal}

P.O. Box 94999

Fort Stellacoom, WA 98494

Phone: (206) 756-2519

Contact: Ronald Hansen

Type of Business: State Hospital

Type of Biomass Fuel: Wood Pellets

Source of Fuel: Purchased, Northwest Pellet Mills

Annual Biomass Fuel Consumed: 12,000 tons

Back-up Fuel: Natural gas

End Use(s): Space heat, kitchen, laundry

Plant Design Capacity: 3 boilers

Heat (million Btu/hr): $28,311,140-77,212,200$ $51,474,800$

Steam: 22,000; 60,000; 22,000lbs/hr@ 130 psig

Electrical Generation (MW): None

Average Plant Output:

Heat (million Btu/hr): $64,343,500$

Steam: 50,000 lbs/hr @ 120 psig (Fall,Winter, Spring)

Electrical Generation (MW): None

Type of Combuster/Furnace: Stoker

Manufacturer: Hoffman

Type of Boiler: Water tube

Boiler Manufacturer: Garet \& Shater; WyattKipper; Puget Sound Machine

Type of Pollution Control: None
Reason for Using Wood Fuel: Emission problems with coal 


\section{Wood Combustion Facilitles}

\section{L.Isting Changes from 1987 Edition}

\section{New LIstlngs}

Colville Indlan Precision Pine Company

Fred Tebb \& Sons, Inc.

Goodyear Nelson Hardwood Lumber Co., Inc.

ITT Rayonier, Inc. - Hoquiam Jeld-Wen Fiber of Washington

Smith Street Mill, Inc.

Strauser Manufacturing

Summit Timber Power House

West Coast Forest Products

Weyerhaeuser

\section{Deletloris}

\section{Boise Cascade}

Howard Manufacturing

Lacey Plywood

Paxport Mills, Inc.

Seattle Door

\section{W.I. Forest Products}

White Swan Lumber

\section{Facllity Name Changes}

\section{Allen Logging Company \\ Daishowa America \\ James River \\ K-Ply inc.}

Omak Wood Products, Inc.

Plum Creek Manufacturing, Inc.

\section{Location}

Omak

Tacoma

\author{
Sedro Woolley \\ Hoquiam \\ White Swan \\ Everett \\ Walla Walla \\ Darringtonn \\ Arlington \\ Cosmopolis
}

\section{Cause}

Locatlon

Closure

Switched to Natural Gas

Closure

Sold

Sold - New facility does

not use biomass

Clocure

Closure

Goldendale

Kent

Lacey

Tacoma

Seattle

Cashmere

Whilie Swan

\section{Former Name}

\section{Location}

Allen Logging \& Vaneer

Crown Zellerbach

Crown Zellerbach

Peninsula Plywood Company

Cavenham Forest Industries

Plum Creek Lumber
Forks

Port Angeles

Camas

Port Angeles

Omak

Colville 


\section{Ethanol, Municipal Solid Waste and Solid Fuel}




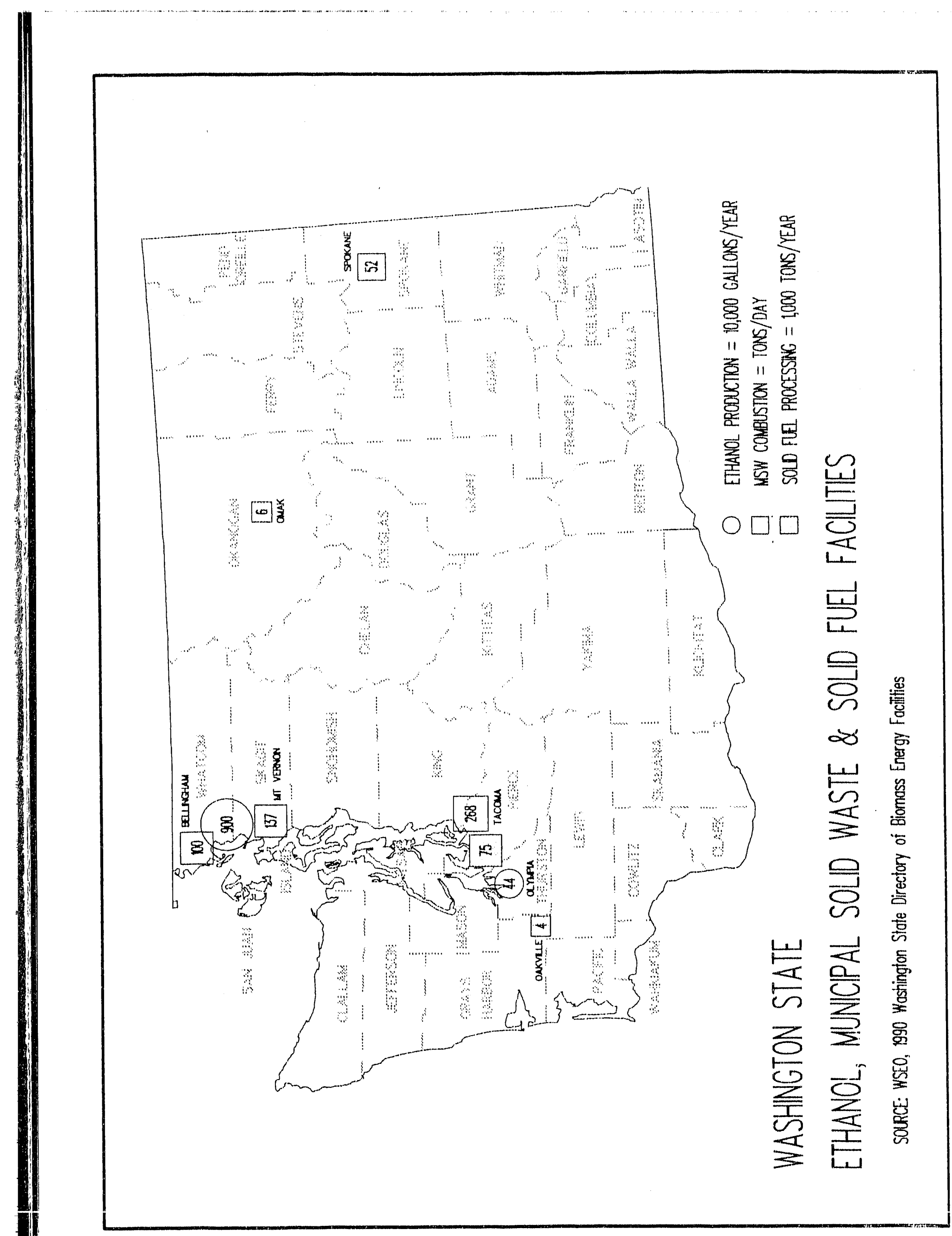




\section{Washlngton State Blomass Summary Tables}

Summary Table 4

\section{Ethanol Production \\ Facilltles Sorted by Output}

\begin{tabular}{|c|c|c|c|c|}
\hline Eacllity Name & Clty & $\begin{array}{l}\text { Plant } \\
\text { Capaclity } \\
\text { Gal./Yr. }\end{array}$ & $\begin{array}{l}\text { Average } \\
\text { Plant } \\
\text { Output } \\
\text { Gal./Yr. }\end{array}$ & $\begin{array}{c}\text { Gross Energy } \\
\text { Equivalent } \\
\text { Million } \\
\text { Btu/Yr." }\end{array}$ \\
\hline $\begin{array}{l}\text { Georgia-Pacific Corportion } \\
\text { Olympla Brewing Company } \\
\text { Total }\end{array}$ & $\begin{array}{l}\text { Bellingham } \\
\text { Olympia }\end{array}$ & $\begin{array}{r}9,000,000 \\
1,000,000 \\
10,000,000\end{array}$ & $\begin{array}{r}9,000,000 \\
435,000 \\
9,435,000\end{array}$ & $\begin{array}{r}761,490 \\
36,805 \\
798,295\end{array}$ \\
\hline
\end{tabular}

"Based on 84,610 Btu per gallon ethanol output
MSW Combustion

Facillties Sorted by MSW Consumption

\begin{tabular}{|c|c|c|c|c|}
\hline Facllity Name & Clty & $\begin{array}{c}\text { Annual MSW } \\
\text { Consumptlon } \\
\text { Tons/Yr. }\end{array}$ & $\begin{array}{c}\text { Average MSW } \\
\text { Consumptlon } \\
\text { Tons/Day }\end{array}$ & $\begin{array}{c}\text { Gross Energy } \\
\text { Equlvalent } \\
\text { Million } \\
\text { Blu/Yr." }\end{array}$ \\
\hline $\begin{array}{l}\text { Tacoma Steam Plant \#2 } \\
\text { Skagit Co. Resource }\end{array}$ & Tacoma & 98,000 & 268 & 980,000 \\
\hline Recovery Facillty & Mount Vernon & 50,000 & 137 & 500,000 \\
\hline $\begin{array}{l}\text { Thermal Reduction Company } \\
\text { Total }\end{array}$ & Bellingham & $\frac{36,500}{184,500}$ & $\frac{100}{505}$ & $\frac{365,000}{1,845,000}$ \\
\hline
\end{tabular}

"Based on annual MSW consumption at 5,000 Btu per pound

Summary Table 6

Solld Fuel Processing

Facllitles Sorted by Plant Output

$\begin{array}{llrrr}\text { Facllity Name } & \text { Clty } & \begin{array}{c}\text { Plant } \\ \text { Deslgn } \\ \text { Capaclty } \\ \text { Tons/Yr. }\end{array} & \begin{array}{c}\text { Average } \\ \text { Plant } \\ \text { Output } \\ \text { Tons/Yr. }\end{array} & \begin{array}{c}\text { Gross Energy } \\ \text { Equivalent } \\ \text { Million } \\ \text { Btu/Yr. }\end{array} \\ \text { Manke Lumber Company } & \text { Tacoma } & 74,880 & 74,880 & 1,272,960 \\ \text { Spokane Pres-To-Logs } & \text { Spokane } & 69,000 & 52,000 & 884,000 \\ \text { CD Pellet Company } & \text { Omak } & 6,000 & 6,000 & 102,000 \\ \text { Wood Pellet Company } & \text { Oakville } & \underline{36,500} & \underline{3,650} & \underline{62,050} \\ \text { Total } & & 186,300 & 135,450 & 2,321,010\end{array}$




\section{Ethanol Production Facilities}

\author{
Georgla-Pacific Corporation \\ P.O. Box 1236 \\ Bellingham, WA 98225 \\ Phone: (206) 733-4410 \\ Contact: Jan Draut
}

Type of Business: Pulp paper, chemicals

Type of Blomass Fuel: Ethanol

Source of Fuel: Spent pulping llquor

Plant Design Capacity:

Gallons per year: 6,000,000@190 proof, 3,000,000@200 proof

Average Plant Output: Gallons per year: 6,000,000@190 proof, 3,000,000@200 proof

\section{Olympla Brewling Company}

P.O. Box 947

Olympia, WA 98507

Phone: (206) 754-5010 or 754-5057

Contact: Paul D. Knight or Larry L. Sidor

Type of Business: Beer

Type of Blomass Fuel: Ethanol

Source of Fuel: Spillage, tank wash outs

Plant Design Capacity:

Gallons per year: 1,000,000@200 proof

Average Plant Output:

Gallons per year: 435,000@200 proof

\section{Municipal Solid Waste Combustion Facilities}

\author{
Skaglt County Resource Recovery \\ Facillty \\ 1200 W Ovenell Street \\ Mount Vernon, WA 98273 \\ Phone: (206) 336.9400 \\ Contact: William H. Ness, P.E.
}

Type of Biomass Fuel: Municipal Solid Waste

Source of Fuel: Skagit County

Arnual Biomass Fuel Consumed: 50,000 tons
End Use(s): Provide electricity to Puget Power

Plant Design Capacity: 178 tons per day Heat (million Btu/hr):

Steam: $40 \mathrm{lbs} / \mathrm{hr} @ 450 \mathrm{psig}$

Electrical Generation (MW): 2.3

Average Plant Output:

Heat (million Btu/hr):

Steam: $40 \mathrm{lbs} / \mathrm{hr} @ 450 \mathrm{psig}$

Electrical Generation (MW): 2.3

Type of Combustor/Furnace: Rotary Kiln

Manufacturer: Tecnitalla

Type of Boiler:

Boller Manufacturer: Zurn

Type of Pollution Control: Acid gas scrubber \& baghouse

Tacoma Steam Plant No. 2

Tacoma Clty Light

P. O. Box 11007

Tacoma, WA 98411

Phone: (206) 593-8336

Contact: Patrick D. McCarty, P.E.

Type of Blomass Fuel: Refuse derived fuel and hog fuel

Source of Fuel: Clty of Tacoma

Annual Biomass Fuel Consumed: 98,000 tons

End Use(s): Electricity

Plant Design Capacity: 3,168 tons RDF/day, 820 tons hog fuel/day, 494 tons coal/day Heat: (million Btu/hr):

Steam: 528,000lbs/hr@382 psig Electrical Generation (MW): $43 \mathrm{MW}$ net

Average Plant Output: 3,169 tons RDF/day, 820 tons hog fuel/day, 494 tons coal/day Heat (million Btu/hr):

Sieam: 528,000 lbs/hr @ 382 psig Electrical Generation (MW): $43 \mathrm{MW}$

Type of Combustor/Furnace: Fluidized bed

Manufacturer: Energy Products of Idaho

Type of Boller:

Boiler Manufacturer:

Type of Pollution Control: Limestone in bed, baghouses 


\section{Thermal Reduction Company}

1524 Slater Road

Bellingham, WA 98226

Phone: (206) 676-0660

Contact: James R. Anderson

Type of Blomass Fuel: Muniolpal Solld Waste

Source of Fuel: City of Bellingham

Annual Blomass Fuel Consumed: 100 tons per day

End Use(s): Electricity

Plant Design Capacity: Heat (million Btu/hr):

Steam: 25,000 lbs/hr@400 psig

Electrical Generation (MW): 2.0

Average Plant Output:

Heat (million Btu/hr):

Steam: 25,000 lbs/hr @ 400 psig

Electrical Generation (MW): 2.0

Type of Combustor/Furnace: Modular Starved Air

Manufacturer: Consumat

Type of Boller: Water tube

Boller Manufacturer:

Type of Pollutlon Control: Electrostatic preclpitator

\section{Solid Fuel Processing Facilities}

\section{Pellet Company}

P. O. Box 3185

Omak, WA 98841

Phone: (509) 826- 2059

Contact: Cherie or Dick Peterson

Type of Feedstock: Shavings

Source of Feedstock: Local mill

Plant Design Capacity: 6,000 tons per year

Average Plant Output: 6,000 tons per year

Type of Products: Wood pellets

Product Storage Capacity: 2,000 tons

\section{Manke Lumber Company}

1717 Marine View Drive

Taooma, Washington 98422

Phone: (206) 572.6252

Contaot: Milton Farsour

Type of Foedstook: Sawdust and Shavings

Source of Feodstock: Local mills

Plant Design Capacity: 240 tons/day $\times 312$ days/year $=74,880$ tons/year

Average Plant Output: 10 tons/hr @ 24 hrs/day $=74,880$ tons/yr

Type of Products: Pellets

Product Storage Capaclty: 6,000 Yons

\section{Spokane Pres-To-Logs}

3124 Flora Road N

Spokane, WA 99216

Phone: (509) 924-2807

Contact: Dan Armstrong

Type of Feedstock: Dry Planner Shavings

Source of Feedstock: Local mills

Plant Design Capacity: $33,0 \mathrm{C} 10$ tons/yr pellets, 36,000 tons/yr logs

Average Plant Output: 26,000 tons/yr pellets, 26,000 ton/yr logs

Type of Products: $1 / 4^{n}$ diameter $1 "$ long pellets (bulk and bags) $8 \#$ logs

Product Storage Capacity: 4,000 tons

\section{Wood Pellet Company}

P.O. Box 87

Oakville, WA 98568

Phone: (206) 273-6982

Contact: Dick Rathvon

Type of Feedstock: Mill residue

Source of Feedstock: Local mills

Plant Design Capaclty: 100 tons per day

Average Plant Output: 10 tons per day

Type of Products: 1/4" pellets, bagged

Product Storage Capacity: 500 tons 


\section{Ethanol Production, MSW Combustion, and}

Solld Fuel Processing Facillties

Llsting Changes from the 1987 Edition

\section{Ethanal Productlon Facllitles}

No additlons or deletlons

Munlclpal Solld Waste Combustlon Facllities

\section{Now Lustlngs}

Skagit County Fesource Recycling Faollity

Tacoma Steam Plant No. 2

No deletions

Solld Fuel Processing Facllitles

New Llstings

CD Pellet

Manke Lumber Company

No deletions

\section{Location}

Mount Vernon

Tacoma

\section{Location}

Omak

Tacoma 


\section{Conversion Table}

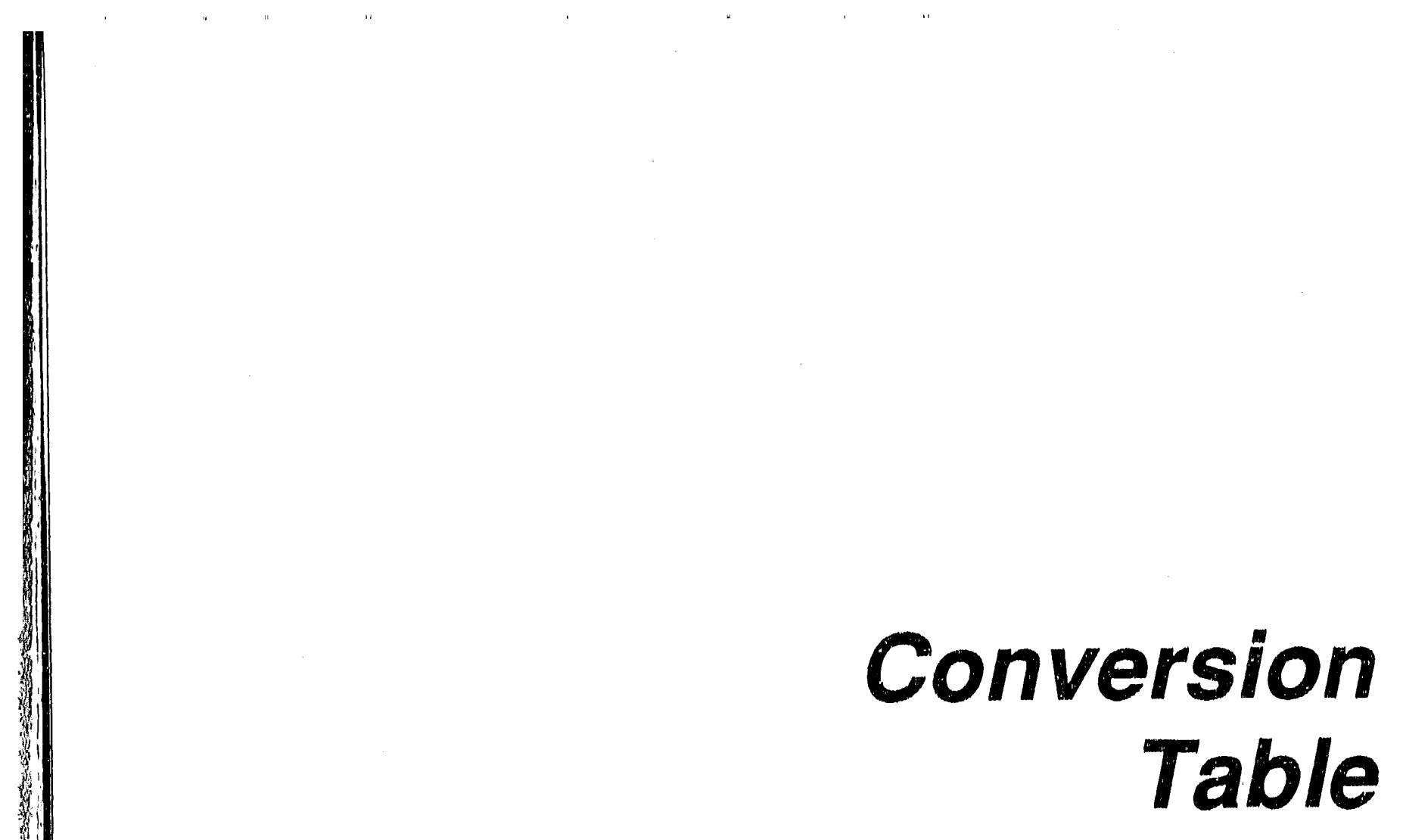




\section{Conversion Table}

Multiply.

Bone Dry Metrlo Ton

Bone Dry Unit

Green Ton

One Gallon Ethanol

Orie Pound Bone Dry Hog Fuel

One Pound MSW

One Ton

Unit
By

1.1023

1.2

(1 - molsture content)

84,610

8,500

5,000

2,000

1.1
To Obtain

Bone Dry Ton

Bone Dry Ton

Bone Dry Ton

Btu

Btu

Btu

Pounds

Bone Dry Tons 
Glossary 


\section{GLOSSARY*}

Anaeroblc: Pertalining to the absence of free oxygen.

Anaeroblc digestion: A blochemloal degradation process that oonverts oomplex organic materials to methane and other by-products.

Bloenergy: The oonversion of the complex carbohydrates In organic matter Into energy, elther by using it direotly as a fuel or by processing it into llquids and gases that are more efflcient.

Blogas: A cumbustlble gas derlved from decomposirig blologlcal waste.

Blomass: Any organlo matter which is avallable to be used as a fuel on a renewable basis Including forest residues, agricultural crops and wastes, wood and wood wastes, animal wastes, livestock operatlon residue, aquatic plants, and muniolpal wastes.

Blomass fuel: Any liquid, solld, or gaseous fuel produced by converslon from blomass.

Boller horsepower: An arbitrary unit in the Engllsh system of measure for the equlvalent of 33,480 Btu gross output or evaporation of 34.5 pounds of water per hour at $212^{\circ} \mathrm{F}$.

Bone dry (oven dry): Having zero percent molsture content. Wood heated in an oven at a constant temperature of $212^{\circ} \mathrm{F}$. or above untll its weight stabillzes.

Bone dry unlt (BDU): A quantity of wood residue which welghs 2,400 pounds at zero percent molsture content.

Brltlsh Thermal Unlt (Btu): The amount of heat requlred to ralse the temperature of one pound of water one degree Fahrenhelt.

Capacity: The maximum instantaneous output of a resource, often expressed in kllowatts or megawatts.

Chlps: Woody materlal cut into short, thin waters, used as a raw material for pulping, fiberboard, or for energy use.

Chlp vans: Special construction bottom dump trucks (grain trucks) or conventional tractor-trailer vans used for haulling pulp chips, mill residues, hog fuel, and other biomass of sinaller piece sizes.

Cogeneration: The technology of producing electric energy and forms of useful energy (thermal or mechanical) for industrial, commerclal, heating, or coollng purposes, through the sequentlal use of an energy source.

Combustion: A chemical process, especially oxidation, accompanied by the production of heat and light. 
Cord: A stack of wood consisting of 128 cubic feet with standard dimensions of $4^{\prime} \times 4^{\prime} \times$ 8 ', including air space and bark; typically 1.2 bone dry tons per cord.

Cublc Foot (CF): $12^{\prime \prime} \times 12^{\prime \prime} \times 12^{\prime \prime}$ or $1^{\prime} \times 1^{\prime} \times 1^{\prime}$.

Dlgester: A vessel in which carbon containing biomass is reacted in water in absence of air to produce biogas.

Dry ton: 2,000 pounds of material dried to a constant weight.

Dutch oven furnace: One of the earliest types of furnaces that is a large, rectangular box, lined on the sides and top with fire brick (refractory) that is commonly used for wood firing. Heat is stored in the refractory and radiated to the conical fuel pile in the center of the furnace.

Ethanol: Ethyl alcohol produced by fermentation and distillation.

Feedstock: Any material which is converted to another form or product.

Fly ash: Small ash particles carried in suspension in combustion gases.

Forest residues: Wasted and unutlized wood in the forest, including logging residue, rough, rotten, and dead trees, and annual mortality.

Fossil fuel: A solid, liquid, or gaseous fuel material formed in the ground by chemical and physical changes in plant and animal residues under high temperature and pressure.

Fuel-cell furnace: A variation of the Dutch oven design, that usually incorporates a primary and secondary combustion chamber. The primary is a vertical refractory-lined cylinder with a grate at the bottom in which combustion is partially completed. Combustion is completed in the secondary cell.

Fuel handling system: The delivered wood fuel is unloaded from vans or trucks and transported to a storage pile or bin. From storage, the wood fuel is conveyed mechanically or pneumatically to the energy conversion system.

Gasification: A chemical or heat process used tc convert a feedstock to a gaseous form.

Gasifler: A device for converting solid fuel into gaseous fuel. In biomass systems, the process is referred to as pyrolytic distillation. See pyrolysis.

Green ton: 2,000 pounds of undried material, e.g., wood residue including moisture content.

Hogged (hog) fuel: Wood waste such as sawdust, planer shavings, and wood chunks normally used for fuel purposes.

Horsepower: Electrical: A measure of time rate of mechanical energy output. The term is usually applied to electric motors to describe maximum output. 1 electrical horsepower $=0.746$ kilowatts $=2,545$ British thermal units per hour. Also see Boiler Horsepower. 
KIlowatt: 1,000 watts, a measure of electrical power.

Logging residues: The unused portions of saw timber and pole timber trees cut or dilled during the harvesting process.

Megawatt (MW): An electrical generation unit of one million watts or 1,000 kllowatts.

Methane: An odorless, colorless, flammable gas that is the primary constituent of natural gas.

MGD: Millon gallons per day.

MIII residue: Wood and bark residues produced in processing lumber.

Molsture content, dry basis (MCDB): Expressed as a percentage of the weight of the oven-dry wood.

$$
\text { MCWB }=\frac{(\text { weight wet sample-weight dry sample })}{\text { weight dry sample }} \times 100
$$

Molsture content, wet basis (MCWB): Expressed as a percentage of the weight of the wood as received.

$$
\text { MCDB }=\frac{\text { (weight wet sample-weight dry sample) }}{\text { weight wet sample }} \times 100
$$

Molsture content (MC): The moisture content is the amount of water contained in the fuel.

Oven dry: See bone dry.

Oven dry ton (ODT): An amount of wood that weighs 2,000 pounds at zero percent moisture content.

Pounds: Pound mass $=\mathrm{A}$ unit of mass equal to 0.454 kilograms.

Pound of steam: One pound mass of water converted to steam.

Power converslon tactors (rate of flow of energy): Watts $=3.413$ British thermal units per hour; kilowatts $=1,000$ watts $=1.341$ horsepower $=3,413$ British thermal units per hour; horsepower $=745.7$ watts, etc.

Psl: Pounds of pressure per square inch.

Pslg: Pounds/force per square inch gauge.

Pyrolysis: A process of burning at less than stoichiometric conditions involving the physical and chemical decomposition of solid organic matter by action of heat in the absence of oxygen. 
RDF: Refuse-derived fuel; the combustible portion of solid waste that has been processed to remove heavier, noncombustible materials.

Renewable energy resource: Any energy resource which has recently originated in the sun, including direct and indirect solar radiation and intermediate solar energy forms such as wind, ocean thermal gradients, ocean currents and waves, hydropower, photovoltaic energy products of photosynthetic processes, organic wastes, and others.

Saturated steam: Steam at the temperature which corresponds to its boiling temperature at the pressure.

SCF: Standard cubic foot.

SCFM: Standard cubic foot per minute.

Scrubber: A device in which combustible or stack gas is cleaned by the spraying of water.

Spreader stoker furnace: Fuel is automatically or mechanically spread across the furnace. A portion is suspension burned, but large pleces fall on a grate.

Steam Turblne: A device for converting energy of high-pressure steam (produced in a boiler) into mechanical power which can then be used to generate electricity.

Turblne: A machine for converting heat energy in steam or high temperature gas into mechanical energy as the high velocity flow of steam or gases passes through successive rows of radial blades fastened to a central shaft.

Unit: 200 cubic feet, bulk measure, used to measure hog fuel, pulp chips, etc. Contains varying amounts of solid material depending on the amount of compaction. It is now more customary to weigh material, correct for moisture, and calculate the number of bone dry tons.

Watt: The common base unit of power in the metric system. 1 kilowatt $=1,000$ watts; 1 $\mathrm{kWh}$ (kilowatt hour) $=$ the amount of power consumed for one hour. 1 megawatt $=$ $1,000,000$ watts.

"Excerpted from: Pacific Northwest and Alaska Bioenergy Program Glossary (1985), and the Department of Energy Blomass Energy Project Development Guidebook. 
Index 


\section{INDEX}

Allen Logging \& V'aneer 36

Allen Logging Company 14, 16, 36

Anacortes Public Works/Operations Division, City of 4,5

Bennett Lumber Products 14, 16

Boise Cascade Corporation - Goldendale 36

Boise Cascade Corporation - Kettle Falls 14, 16, 17

Boise Cascade Corporation - Steilacoom 14, 17

Boise Cascade Corporation - Wallula 14, 18

Boise Cascade Corporation - Yakima 14, 18

Bremerton Wastewater Treatment Plant, Clty of 4,5

Buffelen Woodworking 14, 18

Carkeek Park Treatment Plant 4, 9

Cavenham Forest Industries 36

Central Kitsap Treatment Plant 4, 5

Centralia Wastewater Treatment Plant, Clty of 4,5

CD Pellet Company $38,40,41$

Champion International 14, 19

Chehalls Wastewater Treatment Plant, City of 4,5

Chelan Treatment Plant 4,5

Coastcraft, Incorporated 15, 19

Colville Indian Precision Pine Company 14, 19, 36

Cowlitz Stud Company 14, 19

Crown Zellerbach 36

Daishowa America $16,20,36$

Dayton Wastewater Treatment Plant, City of 4,6

DeGoede Bulb Farms 15, 33

Des Moines Creek Treatment Plant 4, 6, 12

Douglas County Sewer District 4, 6

E. A. Nord Company 16,20

Ellensburg Wastewater Treatment Plant, City of 4,6

Federal Way Water \& Sewer Redondo Treatment Plant 4,6

Fort Vancouver Plywood 16, 20

Fred Tebb \& Sons, Incorporated 17, 20, 36 
Georgia-Pacific Corporation $16,21,38,39$

Goodyear Nelson Hardwood Lumber Company, Incorporated 14, 21, 36

Grand Coulee, Clty of 12

Hardel Mutual Plywood 14, 22

Howard Manufacturing 36

I TT Rayonier, Incorporated - Grays Harbor Dlvision

$14,22,36$

ITT Rayonier, Incorporated - Port Angeles 14, 22

James River 14,23

Jeld-Wen Fiber of Washington 14, 23, 36

Kinnear of Washington 15,23

K-Ply, Inc. $14,24,36$

Lacey Plywood 36

Lacey, Olympla, Tumwater, Thurston County (LOTT) $\quad 4,6$

Layman's Lumber Company 14, 24

Longview Fibre Company 14, 24

Louisiana Pacific Company 14, 25

Manke Lumber Company $38,40,41$

Maple Lane School 15, 33

McCleary Sewage Treatment Plant 4, 7

Metro Alki Treatment Plant 4, 9

Miller Creek Treatment Plant 4, 9, 12

Mount Vernon Sewer Department, Clty of 4,7

Mt. Baker Plywood 14, 25

Newport Wastewater Treatment Plant, City of 4,7

Northwest Hardwoods 14, 25

Oak Harbor, Clty of 4, 7

Oeser Company, The 15,25

Olympia Brewing Company $\mathbf{3 8 , 3 9}$

Omak Wood Products, Incorporated 14, 26, 36

Pacific Wood Treating 14, 26

Pasco Sewage Treatment Plant, City of 4,7

Paxport Mills, Inc. 36

Peninsula Plywood Company 36

Plerce County Chambers Creek Treatment Plant 4, 10

Plum Creek Lumber 36

Plum Creek Manufacturing, Incorporated 26, 36 
Port Angeles, Clty of 4,7

Port Orchard/Kitsap County Sewer Disirict \#5 WTP $4,8,12$

Port Townsend Paper 14, 27

Prosser Wastewater Treatment Facilities, Clty of 4,8

Puget Sound Plywood 14, 27

Pullman Wastewater Treatment Plant 4,8

Puyallup Wastewater Plant 4,8

Rainier State School Sewage Treatment Plant 4,8

Renton Treatment Plant $\mathbf{4 , 8}$

Richland Wastewater Treatment Plant 4, 9

Rlchmond Beach Treatment Plant 4, 9

Salmon Creek Sewage Treatment Plant 4, 9, 12

San Poil Lumber Company 14, 27

Scott Paper 14, 28

Seattle Door 36

Simpson Door Company 14, 28

Simpson Tacoma Kraft Company 14,28

Simpson Timber Company 14, 29

Skagit County Resource Recovery Facillty $38,39,41$

Smith Street Mill, Ino. 15, 29, 36

Southwest Suburban Sewer District 4, 9, 12

Spokane Pres-To-Logs 38, 40

Spokane Wastewater Treatment Plant 4, 10

Stevenson Co-Ply, Inc. 14, 29

Strauser Manufacturing Company 30,36

Summit Timber Power House 30, 36

Sunnyside Wastewater Treatment Plant 4, 10

Tacoma Sewage Treatment Plant-1, City of 4,10

Tacoma Sewage Treatment Plant-2, Clty of 12

Tacoma Sewage Treatment Plant-3, City of 4,11

Tacoma Steam Plant \#2 $38,39,41$

Thermal Reduction Company 34, 40

Toppenish Wastewater Treatment Plant 4, 11

Vaagen Brothers Lumber, Incorporated - Colville 14, 30

Vaagen Brothers Lumber, Incorporated - Ione 14, 31

W.I. Forest Products - Cashmere 36 
W.I. Forest Products - Spokane 14, 33

Walla Walla Wastewater Treatment Plant 4, 11

Washington Soldlers Home \& Colony 15,34

Washington State Penitentiary 14, 34

Washington Water Power Company 14, 34

Wenatchee Water Pollution Control Plant 4, 11

West Coast Forest Products, Incorporated 14, 31, 36

West Point Treatment Plant 4, 10

Western State Hospital 14, 35

Weyerhaeuser Company - Cosmopolis $14,31,36$

Weyerhaeuser Company - Longview 14, 32

Weyerhaeuser Company - Raymond 14, 32

Weyerhaeuser Company - Snoqualmie Falls 14, 32

White Swan Lumber 36

Wood Pellet Company $\mathbf{3 8 , 4 0}$ 


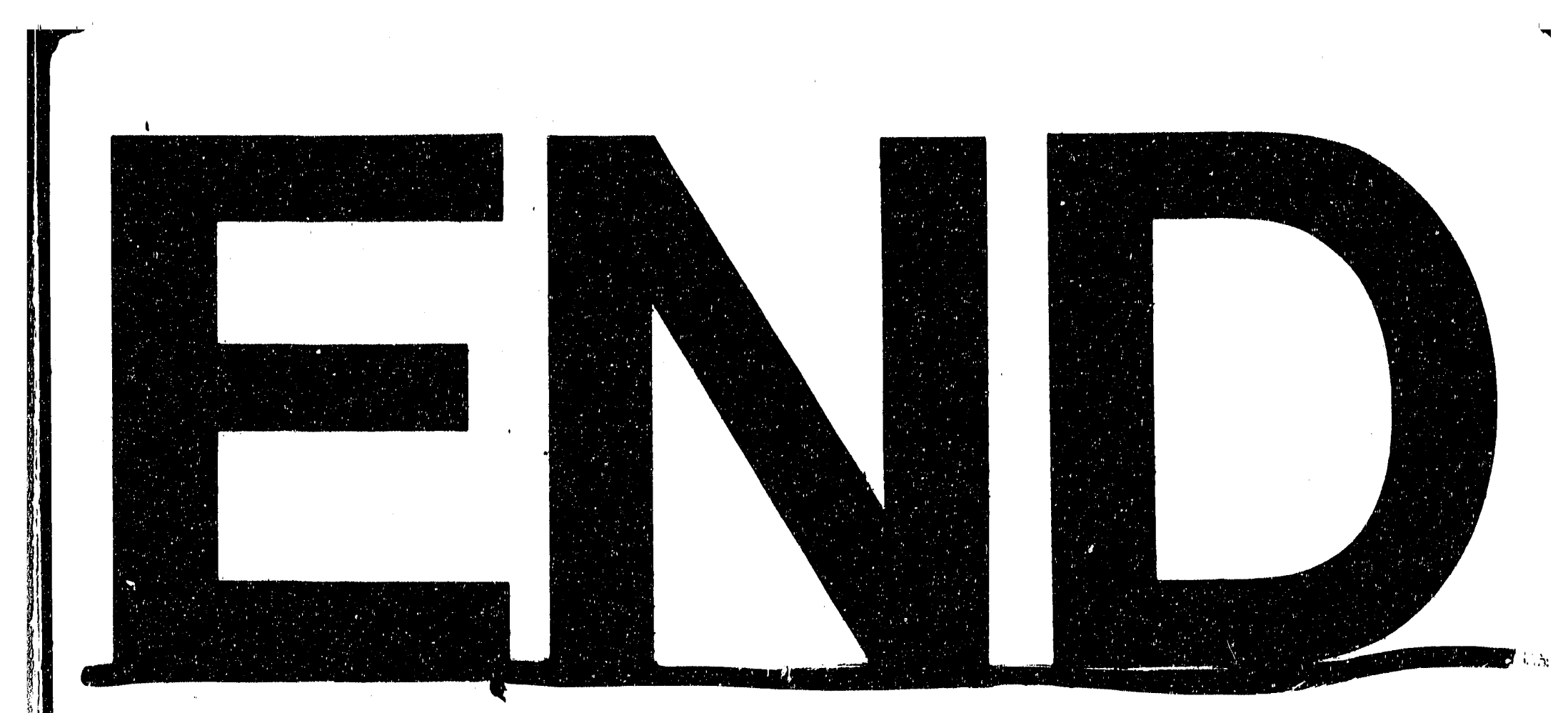


